\title{
Arbitrary Resolution with Two Bead Types Coarse-Grained Strategy and Applications to Protein Recognition
}

\author{
Qinglu Zhong ${ }^{1,2}$ and Guohui $\mathrm{Li}^{1 *}$
}

\begin{abstract}
${ }^{1}$ Laboratory of Molecular Modeling and Design, State Key Laboratory of Molecular Reaction Dynamics, Dalian Institute of Chemical Physics, Chinese Academy of Sciences, Dalian 116023, China

${ }^{2}$ University of Chinese Academy of Sciences, Beijing 100049, China

*Correspondence: ghli@dicp.ac.cn
\end{abstract}




\section{Contents}

- ART CG strategy

$>$ Two-bead-type CG model

$>$ Fitting CG nonbonded parameters

$>$ Optimizing CG positions

$>$ Nonbonded-potential-based mapping

$>$ Fitting $\mathrm{CG}$ bonded parameters

> Detailed results and discussions

$>$ Code availability

$>$ Outlooks

- Simulation details

$>$ Structures

$>$ All-atom simulations

$>$ ART coarse-grained simulations

> MARTINI coarse-grained simulations

$>$ Performances

- Supplementary methods

$>$ Markov state model

$>$ Association rate

$>$ Salt bridge contact

- Supplementary Figures

- Supplementary Tables

- Supplementary References 


\section{ART CG strategy}

\section{Two-bead-type CG model}

We propose a universal two-bead-type CG model, which can be customized to any CG resolutions according to research requirements defined by users. Both bonded beads and nonbonded beads can be mapped to any CG resolution, and the two resolutions can be unrelated. If the molecular backbone is rigid and hard to distort, such as large conjugated porphyrin derivatives, the molecular internal vibrations can be reproduced with fewer bonded CG beads. If the molecular backbone is flexible and has diverse configurations, such as fatty acid derivatives, it must be described by more bonded CG beads with higher resolution. If the molecular property is isotropic, such as methane or fullerene, the molecular peripheral nonbonded potential can be reproduced with fewer (only one for absolutely isotropic) nonbonded CG beads. If the molecular property is anisotropic, such as salicylic acid, it requires not only many nonbonded CG beads to reproduce the disparate nonbonded properties of carboxyl, hydroxyl and phenyl groups respectively, but also more tiled nonbonded CG beads to reproduce the planar property. Thus two types of CG beads with unrelated resolutions are practicable, and even helpful to describe different systems according to different target molecules and purposes.

So how to choose a proper CG resolution? The common way is to divide CG blocks by chemical intuition: for lower resolution, by repeating segments, such as dividing each monomer of a polymer into a CG block; for higher resolution, by important functional groups, such as dividing phosphate, sugar and base groups of a nucleotide into CG blocks; or by user-defined standard, such as the MARTINI ${ }^{1}$ philosophy that manually dividing every four heavy atoms into one CG bead. Since we are emphasizing the arbitrariness of the CG model, we recommend CG blocks be divided by clustering algorithms, which can objectively divide the molecule into any segment. The advantages of subjective division are that it maintains the consistency of chemical properties in the CG model, and ensures that important regions are integrally divided into the specific CG blocks. And the complementary advantages of objective division are that even if an irregular molecule without obvious functional groups can also be automatically divided into any resolutions according to its geometric configuration.

To take respective advantages, our CG model is going to be generated by subjective division before objective division. Researchers first determine a CG resolution, next divide the whole molecule into $X$ segments by chemical intuition, and then automatically divide each segment into $N b$ bonded CG beads (BB) and $N n$ nonbonded CG beads (NB) by clustering algorithm. BB are recommended to be placed at the chemical backbone without moving and fitting, and connected to the next $\mathrm{BB}$ in chemical sequence as CG bonds. NB only require a given resolution, then their positions and nonbonded parameters will be optimized. After fixing the position, each NB will be connected to its closest BB. After that, all bonded parameters will be fitted. For an example to make clear the customized $C G$ resolution: assuming that the resolution is customized that a protein replaced with $3 \mathrm{BB}$ and 2 NB for every 5 residues, $X$ segments correspond to every 5 residues divided manually, $\mathrm{Nb}=3$ means there are $3 \mathrm{BB}$ placed at backbone as recommendation within the 5-residue segment, and $N n=2$ means 2 NB will search 2 appropriate positions within the 5-residue segment by clustering algorithm before optimization (Figure S24). Finally, a two-bead-type CG model is built with an arbitrary CG resolution. 


\section{Fitting CG nonbonded parameters}

Almost all AA force fields adopt Coulomb potential to describe long-range electrostatic interactions and $L J$ 12-6 potential to describe short-range vdW interactions in the nonbonded terms. A pair of nonbonded interaction only depends on the scalar distance between two non-covalently bonded atoms. Coulomb potential is

$$
V_{c}\left(r_{i j}\right)=\frac{1}{4 \pi \varepsilon_{0} \varepsilon_{r}} \frac{q_{i} q_{j}}{r_{i j}}
$$

where $r_{i j}$ is the distance between atom $i$ and atom $j, q_{i}$ and $q_{j}$ are the charges of the two atoms respectively, $\varepsilon_{0}$ is the vacuum dielectric constant and $\varepsilon_{r}$ is the relative dielectric constant. LJ potential is

$$
V_{L J}\left(r_{i j}\right)=4 \varepsilon_{i j}\left(\left(\frac{\sigma_{i j}}{r_{i j}}\right)^{12}-\left(\frac{\sigma_{i j}}{r_{i j}}\right)^{6}\right)
$$

where the potential well depth $\varepsilon_{i j}$ is the combination of $\varepsilon_{i}$ and $\varepsilon_{j}$,

$$
\varepsilon_{i j}=\left(\varepsilon_{i} \varepsilon_{j}\right)^{1 / 2}
$$

and the zero-point distance $\sigma_{i j}$ is the combination of $\sigma_{i}$ and $\sigma_{j}$, but different combination types in different force fields. In Amber or Charmm force field,

$$
\sigma_{i j}=\frac{1}{2}\left(\sigma_{i}+\sigma_{j}\right)
$$

In Gromos or OPLS force field,

$$
\sigma_{i j}=\left(\sigma_{i} \sigma_{j}\right)^{1 / 2}
$$

Whatever, the three nonbonded parameters, $q, \varepsilon$ and $\sigma$, must be included for each atom type in the prevalent AA force fields.

To efficiently perform CG models in the prevalent simulation packages, the nonbonded formalism in the CG force field should be the same as that in AA force field. For fitting CG nonbonded parameters, we need to fit $q, \varepsilon$ and $\sigma$ of each CG bead, and determine a proper combination rule. Consider that if there is a probe atom $p$ at some point outside a molecule, the AA vacuum nonbonded potential sensed by the probe is the sum of nonbonded potential between probe $p$ and each atom $i$ belongs to the molecule.

$$
V_{p}=\sum_{i} \frac{1}{4 \pi \varepsilon_{0}} \frac{q_{i} q_{p}}{r_{i p}}+\sum_{i} 4 \varepsilon_{i p}\left(\left(\frac{\sigma_{i p}}{r_{i p}}\right)^{12}-\left(\frac{\sigma_{i p}}{r_{i p}}\right)^{6}\right)
$$

For the corresponding CG model derived from any CG mapping, the CG vacuum nonbonded potential sensed by the probe is the sum of nonbonded potential between probe $p$ and each CG bead $I$.

$$
V_{p}=\sum_{I} \frac{1}{4 \pi \varepsilon_{0}} \frac{Q_{I} q_{p}}{r_{I p}}+\sum_{I} 4 \varepsilon_{I p}\left(\left(\frac{\sigma_{I p}}{r_{I p}}\right)^{12}-\left(\frac{\sigma_{I p}}{r_{I p}}\right)^{6}\right)
$$


Eq. S7 should be equal to Eq. S6 in an impeccable CG model. Because of the existence of long-range electrostatic and short-range vdW interactions, the ranges of electrostatic and vdW effective distances between the probe and atoms or CG beads are disparate. It can be approximately considered as no coupling between electrostatic and vdW, Coulomb term and LJ term in the Eq. S6 and Eq. $\mathrm{S7}$ are equal respectively.

$$
\begin{gathered}
\sum_{i} \frac{1}{4 \pi \varepsilon_{0}} \frac{q_{i} q_{p}}{r_{i p}}=\sum_{I} \frac{1}{4 \pi \varepsilon_{0}} \frac{Q_{I} q_{p}}{r_{I p}} \\
\sum_{i} 4 \varepsilon_{i p}\left(\left(\frac{\sigma_{i p}}{r_{i p}}\right)^{12}-\left(\frac{\sigma_{i p}}{r_{i p}}\right)^{6}\right)=\sum_{I} 4 \varepsilon_{I p}\left(\left(\frac{\sigma_{I p}}{r_{I p}}\right)^{12}-\left(\frac{\sigma_{I p}}{r_{I p}}\right)^{6}\right)
\end{gathered}
$$

For fitting Coulomb term Eq. $\mathrm{S} 8, q_{p}$ is an independent variable, so let $q_{p}=1 . r_{i p}$ is a known quantity when the coordinates of the molecule and probes are known. Similarly, $R_{i p}$ can be calculated after CG mapping is determined. $q_{i}$ can be looked up in an AA force field. Only $Q_{l}$ is the parameter to be fitted in the Eq. S8. In mathematical methods, if there are enough equations, the parameters in those equations can be derived by regression analysis. So all the charges $Q_{1}$ of $C G$ beads can be solved, provided that probes are enough and widespread around the molecule at a valid distance. For fitting LJ term Eq. S9 with whichever combination rules, $\varepsilon_{p}$ is an independent variable, but $\sigma_{p}$ is not. Thus various values of $\sigma_{p}$ need to be considered in the LJ parameters fitting, because of nonlinear influences on $L J$ potential calculated by different $\sigma$ of probes. In the same way as fitting Coulomb term, $r_{i p}, r_{i p}$ are known and $\varepsilon_{i}, \sigma_{i}$ can be looked up in an AA force field, then all the $U$ parameters $\varepsilon_{l}$ and $\sigma_{l}$ of CG beads can be solved. The difficulty of $L J$ regression is not only computation of sixth-power and twelfth-power terms, but also the two parameters $\varepsilon$ and $\sigma$ are coupled to each other in the equations. The computational complexity of fitting $\sqcup$ parameters is several dozen times higher than that of fitting Coulomb parameters. Computers may not afford this fitting strategy under the circumstances of more CG beads. Typically, $\sqcup$ potential has an effective distance of about $1.2 \mathrm{~nm}$. For macromolecules, most of $r_{i p}$ in Eq. S9 are beyond the effective distance, and few CG beads are involved in the effective distance to a specified probe. In order to reduce the computational expense, we might as well fit $L J$ parameters of each CG bead separately. For single $C G$ bead fitting, the sum of $L J$ potential between one probe and each atom belongs to the $C G$ bead is approximately equal to the $L J$ potential between the probe and the CG bead.

$$
\sum_{i \in I} 4 \varepsilon_{i p}\left(\left(\frac{\sigma_{i p}}{r_{i p}}\right)^{12}-\left(\frac{\sigma_{i p}}{r_{i p}}\right)^{6}\right)=4 \varepsilon_{I p}\left(\left(\frac{\sigma_{I p}}{r_{I p}}\right)^{12}-\left(\frac{\sigma_{I p}}{r_{I p}}\right)^{6}\right)
$$

In this way, only two parameters need to be fitted for each regression, which improves computational efficiency by reducing the requirement of the number of probes to meet regression conditions. However, this simplification may not be suitable for fitting Coulomb parameters, because it is almost impossible that Coulomb term goes beyond its long-range effective distance.

Although $\sqcup$ parameters can be solved out with the above strategy, there will be a remarkable error in the numerical fitting solutions, due to the characteristics of $L J$ function. As if it does not work well using least square method fitting $y=k x^{12}$, but fitting $y^{-12}=k^{\prime} x$ would be much better. The objective of regression algorithms is to minimize the mean-square errors, while $x^{12}$ makes the sampling error extremely sensitive. Therefore, the sample distribution should be as linear as possible so that the regression would be more accurate. Similarly, for Eq. S2, to convert LJ-shaped 
sample distribution to linear distribution, we try to construct the inverse function of $\sqcup$.

$$
V_{L J}^{-1}\left(r_{i j}\right)=r_{i j}\left(V_{L J}\right)= \begin{cases}2^{1 / 6} \sigma_{i j}\left(\left(\frac{V_{L J}}{\varepsilon_{i j}}+1\right)^{1 / 2}+1\right)^{-1 / 6}, & r_{i j} \leqslant 2^{1 / 6} \sigma_{i j} \\ 2^{1 / 6} \sigma_{i j}\left(-\left(\frac{V_{L J}}{\varepsilon_{i j}}+1\right)^{1 / 2}+1\right)^{-1 / 6}, & r_{i j}>2^{1 / 6} \sigma_{i j}\end{cases}
$$

The inverse function has to be expressed as a piecewise function, since $\sqcup$ function is nonmonotonic with positive $\varepsilon$ and $\sigma$. This mathematical manipulation obviously reduces the fitting errors and makes the results more credible.

\section{Optimzing CG positions}

Center of mass is the most common CG mapping because it keeps consistency in momentum space $^{2}$. Nonetheless, it is not necessary to consider momentum consistency as long as there is no velocity in the system before coarse-graining. For instance, Cao and Voth tried to regard center of charge as CG site ${ }^{3}$, which will be used as a comparison later. Center of mass mapping describes dynamical properties well, while center of charge mapping describes electrostatic interactions well (Figure S8). Different mappings have different advantages, so the above mapping strategies may not be optimal to describe $v d W$ interactions. The long-range electrostatic potential is not sensitive to a tiny displacement of a CG site, while the short-range vdW potential is. VdW parameters have the physical significance of describing molecular profiles. Thus it is significant to find an optimal CG site to reproduce molecular peripheral nonbonded potential accurately, especially in the study of molecular recognition, association and dissociation.

For this purpose, we propose a new mapping strategy based on nonbonded potential. It enables CG model to reproduce AA peripheral nonbonded potential, especially vdW, as accurately as possible. In the previous section, $\varepsilon$ and $\sigma$ are regarded as parameters to be fitted under the condition of fixed coordinates $r$. On the other hand, $r$ can also be regarded as parameters to be fitted under the condition of fixed $\varepsilon$ and $\sigma$. This leads to an iterative fitting strategy for both $\sqcup$ parameters and CG coordinates: initialize CG coordinates, then fix coordinates and fit $\mathrm{LJ}$ parameters, next fix $L J$ parameters just derived and fit more proper coordinates, then iterate over and over until it converges. Finally, we achieve a CG model which is the most similar to the AA molecular peripheral nonbonded potential.

\section{Nonbonded-potential-based mapping}

The CG coordinates obtained by the above iterative fitting strategy are uncertain and irregular, so it cannot be expressed as a mapping function corresponding to the AA model. Considering the information transfer between different resolutions in multiscale simulation, there must be a definite mapping relationship between the AA model and the CG model. The mapping matrix $\boldsymbol{M}$ from AA coordinates $r_{i}$ to $C G$ coordinates $R_{l}$ is

$$
\sum_{i} M_{I i} r_{i}=R_{I}
$$

on the condition that 


$$
\sum_{i} M_{I i}=1, \quad \text { for all } \mathrm{I}
$$

where $M_{l i}$ is non-zero only if atom $i$ belongs to CG bead $l$, and the sum of $M_{l i}$ is unitary for all atoms belong to CG bead $I$. Again, if there are enough Eq. S12, $M_{l i}$ can be derived by regression analysis. The minimum number of equations required for linear regression fitting is the number of parameters, non-zero $M_{l i}$, to be fitted. That is to say, the number of conformations required to determine mapping function is inversely proportional to the CG resolution approximately. In order to have enough Eq. S12 to accurately fit the mapping function, we decided to randomly choose multiple conformations from AA simulation trajectories for fitting CG nonbonded parameters and CG coordinates. It also eliminates the influence of different conformations on fitting nonbonded parameters as possible. The mapping function derived above is a mapping strategy that is the most consistent with molecular peripheral nonbonded potential, so we call it nonbonded-potentialbased mapping.

\section{Fitting CG Bonded Parameters}

CG bonded parameters are fitted by Boltzmann inversion ${ }^{4}$ methods, which have been used to fit bonded parameters in a variety of CG models. Briefly, in the canonical ensemble, the degree of freedom $q$ obeys the Boltzmann distribution $P(q) \propto \exp [-\beta U(q)]$, then one can derive its corresponding potential $U(q)=-k_{B} T \ln P(q)$. In this work, one can obtain the distribution $P$ of CG bonded degrees of freedom $q$ from AA trajectories, and then fit CG bonded parameters in the bonded potential function $U$. The degree of freedom can be any bonded terms, like bond, angle, dihedral, even hydrogen bond or $\pi-\pi$ stacking, as long as researchers insist the contacts should be included in the CG simulations. The potential function also can be any bonded function, like harmonic potential, Morse potential, polynomial function or cosine function, as long as it is permitted in the simulation packages. Everything will depend on researchers to adapt to the corresponding research system and simulation environment. The CG model derived from this bonded fitting strategy has an additional benefit that no solvent is required, because the bonded parameters fitted from AA simulation with explicit solvation actually imply the solvation effect. The cost of simulations will be dramatically reduced.

\section{Detailed results and discussions}

We take barnase, a 110-residue protein (PDB 1BRS), as an example and use ART CG strategy to generate its CG force field. The barnase was performed an AA simulation with Amber ff14SB force field ${ }^{5}$ in explicit solvent for $20 \mathrm{~ns}$. A series of AA conformations were obtained from the trajectory. Probes were evenly positioned around each AA conformation. The spatial range, density and $\sigma$ value of the probes can be independently determined by the users. Probes should have an appropriate spatial range, because the $L J$ potential is a short-range interaction sensitive to the distance between the probe and the atom. If the probe is farther away from the molecule, the potential approaches to zero. It does not make sense to fit these worthless probes. The density of probes corresponds to the amount of data to be fitted. The $\sigma$ values of the probes should cover the predicted $\sigma$ range of CG beads as possible. 
In this work, the spatial range of the probes takes a $0.2^{\sim} 0.4 \mathrm{~nm}$ shell outside the molecule with spacing of $0.1 \mathrm{~nm}$ (Figure S1A), $\sigma$ value covers $0.3,0.4,0.5,0.6,0.7 \mathrm{~nm}$. We first take the resolution of one NB per residue as an example. After CG nonbonded parameter fitting through ART CG strategy, the results are as follows. By the inverse LJ fitting method, the fitting error (root-meansquare error, RMSE) of nonbonded parameters of the 110 CG beads is illustrated in Figure S2. CG bead 74 has the smallest fitting error (Figure S2B). As it represents Ala74, which is a small residue deeply buried in the middle of the molecule (Figure S1C), and has only 24 valid probes. Not only that, the distribution of these valid probes is approximately spherical, which is fit for the LJ function form. CG bead 83 has the largest fitting error (Figure S2C). As it represents Arg83, which is a large ribbonlike residue exposed on the molecular surface (Figure S1C), and has nearly 2000 valid probes. Non-spherical distribution of probes also makes greater errors. The fitting errors are mainly between $0.02 \mathrm{~nm}$ to $0.04 \mathrm{~nm}$ (Figure S2A). We select a representative CG bead to explain the advantages of inverse LJ form and the physical significance of its error. The electee is CG bead 64, which represents Pro64. The distances between probes and the CG bead is about $0.3 \sim 1.0 \mathrm{~nm}$. The true $U$ potential for each probe is dotted in the left panel of Figure S2D. The objective is to determine the nonbonded parameters $\varepsilon$ and $\sigma$ subject to the minimum error between real potentials and fitting potentials. Inverse $L J$ function transforms the $L J$ potential to be fitted into the distance between the probe and the CG bead to be fitted. As a result, the objective is altered to determine the nonbonded parameters subject to the minimum error between real distances and distances corresponding to their $\sqcup$ potentials to be fitted. Therefore, the problem becomes a pure linear regression. The fitting error means the difference between the fitting distance and the real distance. The RMSE around $0.03 \mathrm{~nm}$ (Figure S2A) means that the average deviation of LJ potential of all probes is about $0.03 \mathrm{~nm}$ from the CG sites. The solid lines in the left panel of Figure S2D are the $U$ potential curves to the parameters derived by inverse $L$ fitting. Some probes are closer to the CG bead but have lower $\amalg$ potential values while some are farther from the CG bead but have higher $\amalg$ potential values (stars in left panel of Figure S2D). In the LJ potential representation, tens of $\mathrm{kJ} / \mathrm{mol}$ deviations are between these real potentials and the fitting curve, which seems a reasonable fitting instead. If fitting directly rather than by inverse $L$, the small amount of large positive potential values will be given priority to, while a large amount of small negative potential values accuracy will be neglected. Inverse $L$ fitting, however, eliminates the problem of maldistribution of data that all the data are converted to be linear (right panel of Figure S2D). After fitting LJ parameters for each CG bead, interactions between the whole CG molecule and each probe can be calculated. Compared with AA LJ potentials, similarly transforming non-linear data to linear data by inverse $L J$ function, the total RMSE in inverse $U$ scale is about $0.033 \mathrm{~nm}$, linear correlation coefficient is about 0.82 , about $50 \%$ of the absolute errors are within $0.002 \mathrm{~nm}$ and about $80 \%$ of those are within $0.02 \mathrm{~nm}$ (Figure S3).

When CG LJ parameters are optimized, CG positions are also optimized through stepwise iterations to error convergence (Figure S4). Among the iterations, CG LJ parameters are optimized by fixed CG positions of the previous step in odd steps, CG positions are optimized by fixed CG LJ parameters of the previous step in even steps. Interestingly, it is usually slightly better to finish iterating at even steps after CG position optimization. With iterations, the fitting error (RMSE) gradually decreases and converges, the correlation coefficient and the ratio of error less than $0.02 \mathrm{~nm}$ gradually increases and converges. Since ART is a user-oriented strategy, the convergence criteria can be determined by users. In this work, we defined the convergence criteria as that RMSE for even step 
is no longer reduced. For this case, there is a good convergency after iterations over 12 steps. Maybe someone thinks that the error after a 2-step iteration is already acceptable, and then one can use a 2-step iteration to save computational resources when optimizing a similar system after. Different molecules may have different convergencies or convergence steps, researchers need to explore and develop their own fitting strategies to balance errors and computational consuming. In general, the smaller the size, the faster the convergence and the fewer iterative steps required. In this work, to keep consistent in each application, this process is always achieved by iterations over 12 steps, which has been checked to be sufficient to satisfy convergence.

Nonbonded-potential-based (NPB) mapping function can be fitted from the optimal CG positions above and corresponding AA conformations. Then, corresponding CG conformations can be mapped from the AA conformations by the NPB mapping function. Mapping function fitting errors, which are the deviations between the optimal CG positions and the NPB mapping CG conformations, are mainly within $0.05 \mathrm{~nm}$ (Figure S7). NPB mapping is closer to the AA dynamic properties compared with classical center-of-mass mapping. Comparing the AA trajectory with its corresponding center-of-mass mapping CG trajectory and NPB mapping CG trajectory, root-meansquare deviations (RMSD) of the three trajectories to the first frame are roughly coincident (Figure $\mathrm{S} 5 \mathrm{~A})$. It shows that the two mapping functions are both feasible. Further, for more detailed analysis, more than $99 \%$ of NPB mapping RMSD values are closer to AA RMSD values compared with centerof-mass mapping to AA. It is similar for the analysis of root-mean-square fluctuation (RMSF) of the three trajectories (Figure S5B). More than 74\% of NPB mapping RMSF values are closer to AA RMSF values compared with center-of-mass mapping to AA. As a result, NPB mapping is not only better in internal dynamic properties than convinced center-of-mass mapping, but also more accurate for molecular peripheral nonbonded potential profiles.

Then, we repeated the fitting process five times independently to explore whether the parallel results are similar and whether a single trial can be reliable. Relative variances of $110 \mathrm{CG} L$ parameters $\sigma$ are around 1\%, and those of $\varepsilon$ are around 3.5\% among five independent trials (Figure S6). The most erratic bead is commonsensible large non-spherical Tyr 97 , which has $4 \%$ relative variance for $\sigma$ and $16 \%$ for $\varepsilon$. Mapping errors of all beads are within $0.05 \mathrm{~nm}$ with tiny variance except a conspicuous lle88 (Figure 57). Because lle88 is almost buried in the most middle of barnase (Figure S1C), and is one with the fewest valid probes among 110 beads. It is a common problem that a CG bead deeply buried in the molecule is subjected to fewer valid probes, which are just outside the molecule. Fewer probes, fewer samplings, then LJ potential and mapping may be unreliable. But it is not a big deal that the deeply buried beads are hardly involved in intermolecular interactions. Therefore repeated results are similar. Even if someone does not believe a single trial is reliable, it is also advisable to select the minimum error one among the multiple trials.

Theoretically, these CG nonbonded parameters are completely dependent on the limited AA conformations for fitting, but it is uncertain whether it is suitable for other conformations. From experience, parameters derived from different kinds of conformations may have a universal application. For this reason, we explored the relationship between the numbers of conformations to be fitted and fitting errors, universality of derived parameters, amounts of computation. 20-ns AA trajectory has 2000 frames. We randomly chose $N$ frames to fit CG nonbonded parameters what is similar to training sets in machine learning; and use the rest $2000-N$ frames to calculate errors 
between AA nonbonded potential and corresponding CG nonbonded potential according to the training parameters and mapping function what is similar to testing sets in machine learning. Training errors only show the quality of fitting strategy in maths, while testing errors truly represent the applicability of the CG model. With the increase of the number of training frames, both the training error and the testing error gradually decrease and converge, while the computational consumption increases (Figure S10). As a result, using 32 frames to fit CG nonbonded parameters seems to have higher cost performance.

Before fitting Coulomb potential, we have to adjust the probes to suit for long-range interactions. In this work, the spatial range of the probes for Coulomb fitting takes a $0.4 \sim 1.5 \mathrm{~nm}$ shell outside the molecule with spacing of $0.25 \mathrm{~nm}$ (Figure S1B). With the adjusted probes, the results of fitting Coulomb potential are as follows. There are more than 10,000 probes per frame for fitting Coulomb parameters. The error between the fitting Coulomb potential and the real Coulomb potential exerted to each probe is about $8.0 \mathrm{~kJ} / \mathrm{mol}$, and the correlation coefficient is about 0.987 (Figure S8D). However, in the common bottom-up CG force fields, CG charge is simply expressed as the sum of AA charges $(\mathrm{SOC})$ belong to the corresponding $C G$ bead, rather than derived by complicated fitting. Moreover, CG positions or mapping functions also affect CG Coulomb potentials. To characterize the superiority of $\mathrm{CG}$ charges optimized by regression, we compared fitting errors with SOC errors in three different mapping functions, which are center-of-mass mapping (Figure S8A), center-of-charge mapping (Figure S8B) and nonbonded-potential-based mapping without optimizing charges (Figure $\mathrm{S8C}$ ). In the center-of-charge mapping ${ }^{3}$, all positive atoms belong to a CG block are mapped into one positive CG bead weighted by their absolutely partial charge values, and so are negative atoms. Each CG block is described by one positive bead and one negative bead. So the center-of-charge mapping has higher resolution and smaller error than other mapping functions. The errors of center-of-mass mapping and potential-based mapping are almost the same. It is illustrated that CG positions are not sensitive to long-range interactions. That is why we need to fit $\mathrm{LJ}$ parameters at first and determine the LJ-potential-based mapping function before optimizing Coulomb parameters. For the same potential-based mapping, compared with the simple summation, the error after optimizing is significantly reduced. Similar to fitting LJ parameters, multiple trials of fitting Coulomb parameters were repeated independently. Variances of 110 CG Coulomb parameters $q$ are around 0.05 e (Figure S9).

The result of fitting Coulomb parameters with multiple conformations is similar to that of fitting $\mathrm{LJ}$ parameters, that fitting error of testing set decreases and converges after 32 training frames as the number of training frames increases (Figure S10). But for different molecules, the number of training frames with the highest cost performance may be different. If researchers are lack of experience, researchers can also do such tests to find an appropriate number of training frames. Tens of training frames are usually appropriate. For a protein with about 100 residues like barnase, we consider the balance between fitting error with 32 training frames and its computational consumption is acceptable. For this reason, all of the nonbonded fittings will be with 32 training frames in this work.

We just have concluded the cost-effective fitting scheme when CG resolution is one nonbonded bead per residue. Next, we use the same fitting scheme to explore the fitting accuracy in different CG resolutions. Theoretically, the resolution can be arbitrary in our CG model. For proteins, the resolution is expressed by Nn NB per Nr residues. For the above example, CG resolution is one NB 
per residue, i.e. $N n=1$ and $N r=1$ (abbreviated as $N n / N r=1 / 1$ ). For higher resolution, we will try multiple beads per residue, such as $2 / 1,3 / 1$. For lower resolution, we will try one bead per multiple residues, such as $1 / 2,1 / 3,1 / 4,1 / 5$. Although $N n=1$ or $N r=1$ is the recommended resolution for significantly chemical intuition, we will try to test a variety of resolutions in which neither $\mathrm{Nn}$ nor Nr equals to 1 , such as from higher to lower resolution 3/2,2/3,2/5. In addition, the whole protein molecule can also be regarded as one CG block, 110 beads in the whole barnase has the same resolution as one bead per residue, i.e. $110 / 110=1 / 1$, but the results may be different. To explore the difference between treating the whole protein and treating only a few residues as one CG block at the same $C G$ resolution, we will also try 330/110, 220/110, 165/110, 110/110, 74/110, 55/110, $44 / 110,37 / 110,28 / 110,22 / 110$, which correspond to the CG resolution above. As expected, the lower the CG resolution, the larger the fitting error and the worse the fitting correlation (Figure S11 and Table S1). The fitting accuracy of the training set tends to converge, with the CG resolution higher than 1/1, for both vdW and electrostatic interactions. As the CG resolution decreases, the fitting correlation of vdW interactions becomes significantly worse. It means that lower CG resolution is not sufficient to reproduce molecular peripheral $L J$ potential profiles accurately. The size of CG bead is already beyond the range of atomic short-range interactions. It also explains why $\mathrm{LJ}$ potential is rarely applied in the existing CG force field with lower resolution. However, the fitting correlation of electrostatic interactions is still high that is more than 0.9 at lower CG resolutions. It is inferred that long-range nonbonded potential is more suitable to describe a CG force field with lower resolution. Moreover, if the whole protein is regarded as one CG block, the fitting error of electrostatic interactions that are not sensitive to CG positions is unexpectedly smaller at lower CG resolutions. It indicates that when building a CG force field with low resolution, it is not reasonable to use a bead instead of several adjacent residues, which is almost impossible to distribute spherically. Therefore, we believe that one NB per residue (1/1) is the optimal resolution for building protein CG models.

Each $\mathrm{BB}$ and its neighbor $\mathrm{BB}$ in $\mathrm{AA}$ topology are connected by a $\mathrm{CG}$ bond ( $\mathrm{BB}$ bond) for describing CG molecular backbone. Each NB and its closest BB are connected by a CG bond (NB bond) for describing local flexibility of sidechains. For 110 -residue barnase, if each $\alpha$-carbon site is regarded as one BB and each residue is replaced by one NB, there are 109 BB bonds and 110 NB bonds in total. Moreover, hydrogen bonds that maintain secondary and tertiary structures need to be taken in consideration. Each CG bead pairs that represent donor and acceptor are connected by a CG bond ( $\mathrm{HB}$ bond) for maintaining spatial structures. For other molecules, there may be other interactions that must be considered as a CG bond, such as disulfide bonds or coordination bonds. Pseudo CG angles and dihedrals are topologically determined by these CG bonds. In this work, 2000 frames in the 20-ns AA trajectory were used for fitting bonded parameters by Boltzmann inversion method. BB bonds and NB bonds are fitted as harmonic bond potential, and fitting errors are distributed around 0.8 and $1.2 \mathrm{~kJ} / \mathrm{mol}$, respectively (Figure S12A). HB bonds are fitted as Morse potential, which is better for describing hydrogen bond breaking and formation, and fitting errors are distributed around $1.5 \mathrm{~kJ} / \mathrm{mol}$ (Figure S12A). CG angles are fitted as Quartic potential, which is available in GROMACS $^{6}$ and better to describe asymmetric double-well potential than harmonic potential for non-uniformly distributed CG angles, and fitting errors are distributed around 1.1 $\mathrm{kJ} / \mathrm{mol}$ (Figure S12B). CG dihedrals are fitted as proper dihedral potential, and fitting errors are distributed around $1.2 \mathrm{~kJ} / \mathrm{mol}$ (Figure S12C). The representative fitting details for each CG bond type are illustrated in Figure S13. Actually in the program, researchers can fit any bond type by any 
potential functional form, as long as the functional form is suitable for describing the bond type and permitted in the simulation package.

\section{Code availability}

ART CG strategy will be implemented by a user-friendly Python script package. All CG optimization and input files for GROMACS are generated by a series of Python scripts with quick and easy operation for users. These scripts and readable and extendible for users or our further maintenance and improvements. The package is available upon request and going to be released to our homepage later.

\section{Outlooks}

Other types of molecular recognition. In this work, we only demonstrated the effectiveness of the ART strategy for protein recognition. Nevertheless, molecular recognitions of other types of molecules, nucleic acids, lipids and organic small molecules, are also crucial in life processes. Although the ART strategy can be applied to any molecule in theory, the optimum CG resolution is different for different types of molecules. For other types of molecules, it still needs further studies to explore the appropriate CG resolution, such as the results of Figure S10 and S11. Other types of molecular recognition are in our further research plan and under exploration. More Suggestions from researchers are expected.

Large conformational changes. In this work, we mainly focus on the recognition of proteins without large conformational change. However, there are more complicated molecular recognitions that are required to undergo large conformational changes. ART is a system-specific CG model, rather than a universal CG model. ART has the ability to learn everything in the AA training set something like machine learning, while conformations far beyond the training set cannot be created by itself (at least currently). If there exists a large conformational change in the training set, there will record information on the conformational change in the derived CG model. As a result, short AA simulation for CG optimization may not be sufficient for large conformational changes. For this challenge, we will be necessary to consider multi-scale strategy to readjust AA accurate kinetics and CG high-performance.

Multi-scale simulations. To balance AA accurancy with CG high-performance, multi-scale simulation is an inevitable topic for advanced CG models. ART strategy has been reserved compatibility with AA in many aspects, such as the consistency of any AA and its derived CG force field, accurate mapping function for multi-scale conversion. Combining AA and CG, both two resolutions merged in one system and two resolutions converted back and forth, will be done in the further studies. A multi-scale strategy extended from ART is in our further research plan and under exploration.

Practical applications. ART CG simulations can capture the recognition kinetics at nanoscale, which is hard for traditional AA simulations. It achieved completely stochastic and spontaneous protein recognitions in this work. But the computational comsumption will still be a limitation on more complicated recognitions with this simulation scheme. ART with enhanced sampling method has 
the potential to provide a glimpse of simulation view in more complicated recognition processes. Then some novel regularity may be inferred from few unprecedented observations. Although the regularity may have some intrinsic physiological significance, it has not been fully confirmed and need further experimental verification. 


\section{Simulation details}

\section{Structures}

All the molecular structures involved in this work were listed in Table S2. The mutations and the missing residues or missing atoms of PDB structures were completed using MODELLER ${ }^{7}$.

\section{All-atom simulations}

Each molecule was respectively positioned into a cubic box extended $1 \mathrm{~nm}$ from the solute surface and dissolved with TIP3P8 waters. $0.15 \mathrm{M}$ chloride and sodium ions were added to neutralize the net charge of the system. All AA simulations were performed using GROMACS 6 with ff $14 \mathrm{SB}^{5}$ force field. The system was subjected to energy minimization using the steepest descent algorithm, then gradually heated to $300 \mathrm{~K}$ under NVT ensemble and equilibrated under NPT ensemble. The step size of leapfrog verlet integrator was set to $2 \mathrm{fs}$. Particle-Mesh Ewald ${ }^{9}$ method was utilized to treat the electrostatic interactions with cutoff distance of $1.2 \mathrm{~nm}$. The same cutoff value was chosen for treating the van der Waals interactions. LINCS $^{10}$ algorithm was used to constrain covalent bonds involving hydrogen atoms. The Nose-Hoover ${ }^{11-12}$ Thermostat was applied to couple temperature at $300 \mathrm{~K}$ in the system every 2 ps. The Parrinello-Rahman ${ }^{13}$ Barostat was used to control pressure in the system at 1 bar. Each production run was performed 20 ns for generating respective ART CG force field.

\section{ART coarse-grained simulations}

The initial CG conformation of each system was described in the Results of main text. All ART CG simulations were performed using GROMACS with ART CG force field. The system was subjected to energy minimization using the steepest descent algorithm, then gradually heated to $300 \mathrm{~K}$ under NVT ensemble. The step size of Langevin integrator was set to $10 \mathrm{fs}$. The temperature was controlled at $300 \mathrm{~K}$ using V-rescale ${ }^{14}$ thermostat with a coupling constant of $10 \mathrm{fs}$. The relative dielectric constant of Coulomb interaction with reaction-field ${ }^{15}$ was modified from 16 to 80 gradually within $2.4 \mathrm{~nm}$, and fixed 80 beyond $2.4 \mathrm{~nm}$. The cutoff of van der Waals interactions was set to $2.4 \mathrm{~nm}$.

\section{MARTINI coarse-grained simulations}

Each molecule was coarse-grained to MARTINI ${ }^{1}$ model, where the topology and force field of proteins were derived by Martinize script. The scheme of generating initial CG conformation was the same as ART CG simulations. The system was solvated by MARTINI water and neutralized by MARTINI chloride and sodium ions. All simulations were performed using GROMACS with MARTINI force field. The system was subjected to energy minimization using the steepest descent algorithm, then gradually heated to $300 \mathrm{~K}$ under NVT ensemble and equilibrated under NPT ensemble. The step size of leapfrog verlet integrator was set to $30 \mathrm{fs}$. Reaction-field method with a relative dielectric constant of 15 was utilized to treat electrostatic interactions with cutoff distance of 1.1 $\mathrm{nm}$. The same cutoff value was chosen for treating van der Waals interactions. The temperature was kept $300 \mathrm{~K}$ using the V-rescale thermostat with a coupling constant of $1 \mathrm{ps}$. The pressure was isotropically coupled to 1 bar using Parrinello-Rahman barostat with a coupling constant of 12 ps and compressibility of $3 \times 10^{-4}$ bar $^{-1}$. 


\section{Performances}

Take barnase-barstar system as an example, the performances of AA, ART and MARTINI simulations under different processor conditions were listed in Table S3. It should be noted that performance of ART decreases as the number of CG beads increases, because ART is an implicit solvation model. While performance of AA or MARTINI decreases as the size of simulation box increases, rather than the size of solutes, because AA or MARTINI model is filled with large number of solvents. For ART CG simulations, CPU-only is more efficient that ART can be 1,000 times faster than AA simulation under the same condition (Table S3). If GPU is used by GROMACS, nonbonded interactions are computed by GPU and bonded interactions are computed by CPU. When the number of particles is small, GPU computing nonbonded interactions will be a rate-limiting step, then GPU slower than CPU may happen. As a result, different speedup is for different system on different platform. It is a conservative estimation that ART is at least 100 times faster than AA simulations. 


\section{Supplementary methods}

\section{Markov state model}

Feature selection for Markov state model is only residue-residue contact matrix between barnase and barstar. The minimum CG distance between each residue-residue pair $<1 \mathrm{~nm}$ is defined as contact, otherwise non-contact. For each complex conformation, there is one contact matrix; for all trajectories, there are 1 million matrices. Dimensionality reduction computes the seven lowest linear combination of these matrix by VAMP ${ }^{16}$ method with lagtime $100 \mathrm{~ns}$. This space is discretized to 2000 using k-means clustering. All the trajectories are used to estimate the Hidden Markov Model ${ }^{17}$ with lagtime $80 \mathrm{~ns}$. Finally, 14 metastable states and transition rates between each other are estimated. All the Markov procedures are implemented by pyEMMA ${ }^{18}$.

\section{Association rate}

The association rate, $k_{\text {on }}$, is computed by the Smoluchowski rate of hitting a sphere of radius $b$, multiplied by the probability of proceeding to the bound state, rather than dissociating towards infinity, $f_{\infty}$,

$$
k_{\mathrm{on}}=4 \pi D b f_{\infty}
$$

The diffusion constant is determined as $D=D_{1}+D_{2}$, where $D_{1}$ and $D_{2}$ are computed using Einstein Relation, $\left\langle\boldsymbol{x}^{2}\right\rangle=6 \mathrm{Dt}$, according to the independent CG trajectory of the two molecules, respectively. The sphere to which association events occur is defined by $b=4 \mathrm{~nm}$ according to the size of the two molecules. For every time a trajectory enters the $b$-sphere, it is counted how many of them next proceed to an associated state, $n_{\text {associate, }}$ or escape towards infinity, $n_{\text {escape }}$. We define an outside sphere with a radius of $q=5.5 \mathrm{~nm}$, then for every time the trajectory escapes to this sphere, instead of one escape event, only $p_{\text {escape }}=4 \pi D b / q$ escape events are counted. We obtain the binding probability,

$$
f_{\infty}=\frac{n_{\text {associate }}}{n_{\text {associate }}+n_{\text {escape }} p_{\text {escape }}}
$$

For our aggregate 1-ms CG trajectories, we figured out $D_{\text {barnase }}=4.2 \mathrm{~nm}^{2} \mu \mathrm{s}^{-1}, D_{\text {barstar }}=6.3$ $\mathrm{nm}^{2} \mu \mathrm{s}^{-1}, f_{\infty}=0.8916$, so $k_{\text {on }}=2.8 \times 10^{8} \mathrm{M}^{-1} \mathrm{~s}^{-1}$.

\section{Salt bridge contact}

We define a Rational function to make contact continuous from 0 to 1 .

$$
R(r)=\frac{1-\left(\frac{r-d_{0}}{r_{0}}\right)^{6}}{1-\left(\frac{r-d_{0}}{r_{0}}\right)^{12}}
$$

For each pair of salt bridge, $r$ is the distance in each conformation, $r_{0}$ is the reference distance in the experimental structure, $d_{0}$ is set to $0.4 \mathrm{~nm}$. So each salt bridge contact is approximately equal to 1 for each native interface. For a native trimer including three interfaces, each salt bridge contact 
should be 3 . But 3 is not the possible maximum. For K320-E325 as an example, there are three protomers, A, B, C; so there are six possible pairs of salt bridge, A320-B325, B320-C325, C320-A325, A320-C325, C320-B325, B320-A325. The maximum contact is 6 in theory. 


\section{Supplementary Figures}

A

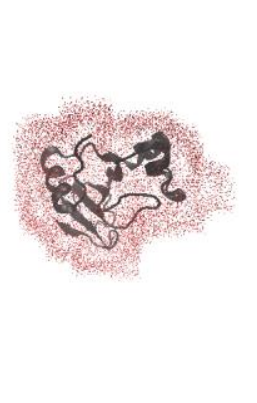

B

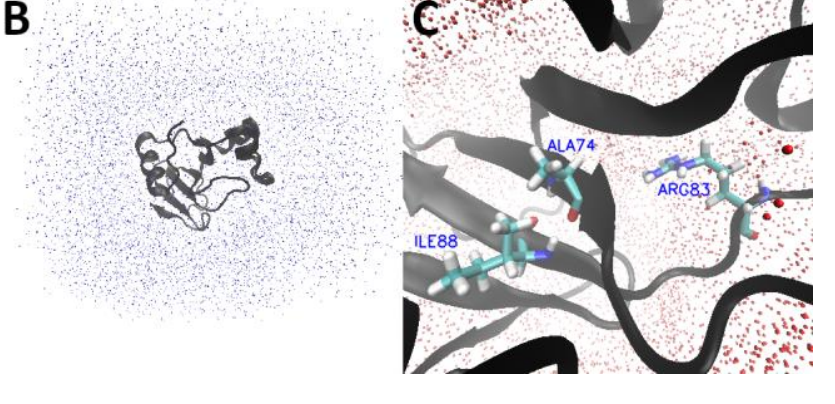

Figure S1. The probes for fitting CG nonbonded parameters. (A) The spatial range of the probes takes a $0.2^{\sim} 0.4 \mathrm{~nm}$ shell outside the barnase for fitting short-range vdW interactions. (B) The spatial range of the probes takes a $0.4 \sim 1.5 \mathrm{~nm}$ shell outside the barnase for fitting long-range electrostatic interactions. (C) Inside-out view of (A), highlight Ala74 and lle88 deeply buried in the barnase and Arg83 exposed on the barnase surface. Black ribbons for barnase, red dots for vdW probes and blue dots for electrostatic probes. 
A

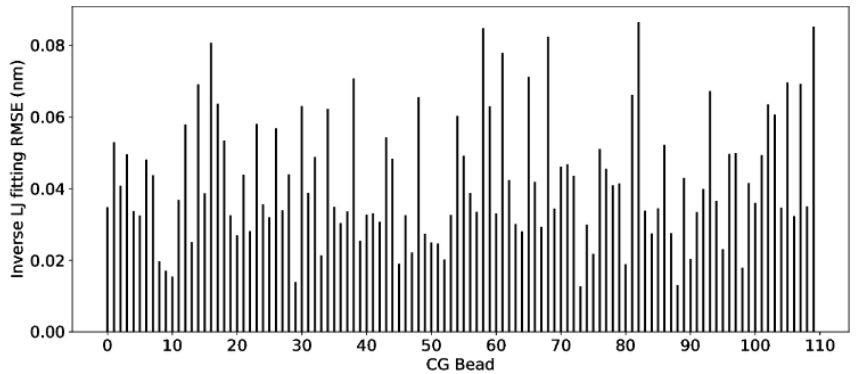

B

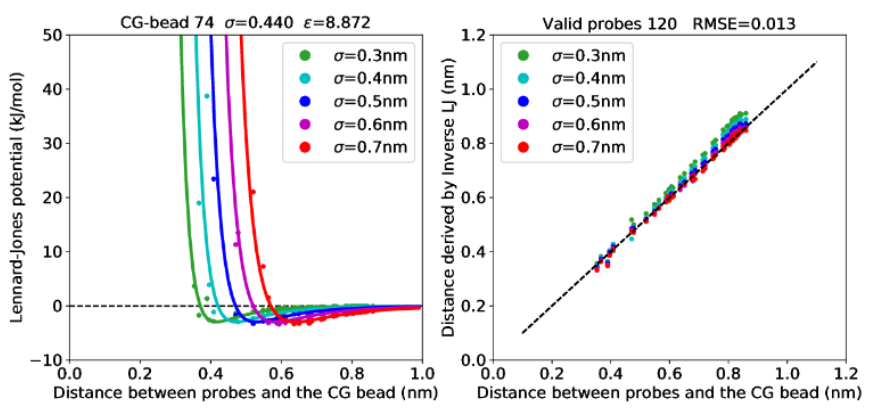

C

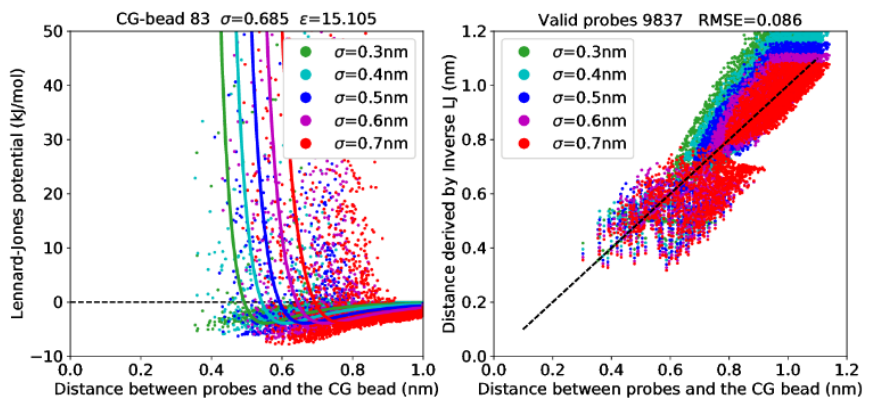

D

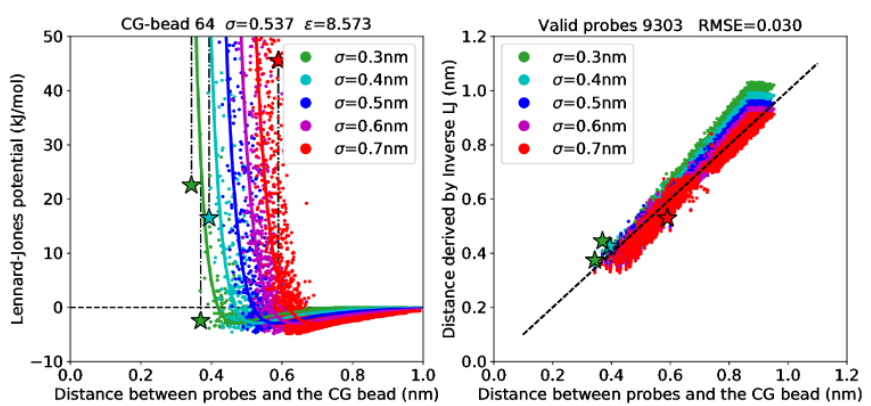

Figure S2. The results of fitting $L$ parameters for each CG bead. (A) The RMSE of inverse $\sqcup$ fitting for each CG bead. (B-D) For each CG bead, the corresponding AA $L J$ potentials detected by its valid probes are dotted in the left panel, the AA inverse $L J$ potentials are dotted in the right panel. $\mathrm{LJ}$ parameters $\varepsilon$ and $\sigma$ of the CG bead are fitted by linear regression. The fitting error is illustrated in (A). CG LJ potential curve of fitted $\varepsilon$ and $\sigma$ is plotted in the left panel. Each probe has five $\sigma$ values, $0.3,0.4,0.5,0.6$ and $0.7 \mathrm{~nm}$, which are colored in green, cyan, blue, magenta and red. (B) CG bead 74 has the minimum fitting error. (C) CG bead 83 has the maximum fitting error. (D) CG bead 64 represents the general fitting accuracy. Stars and black dash-dot reference lines are examples for illustrating the superiority of inverse LJ fitting. 


\section{A}
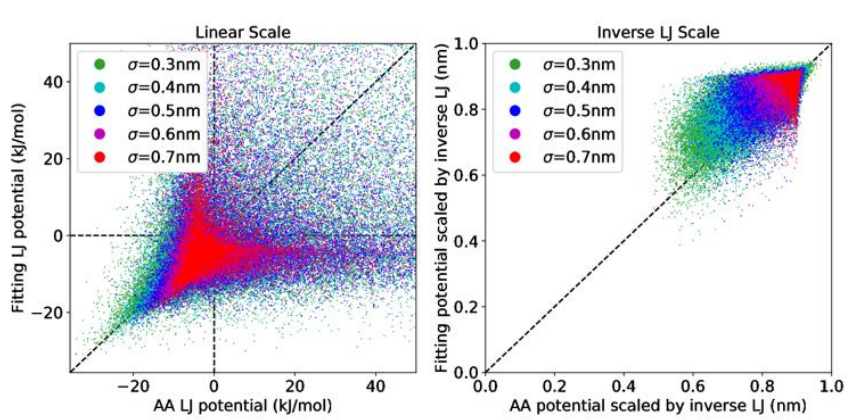

B

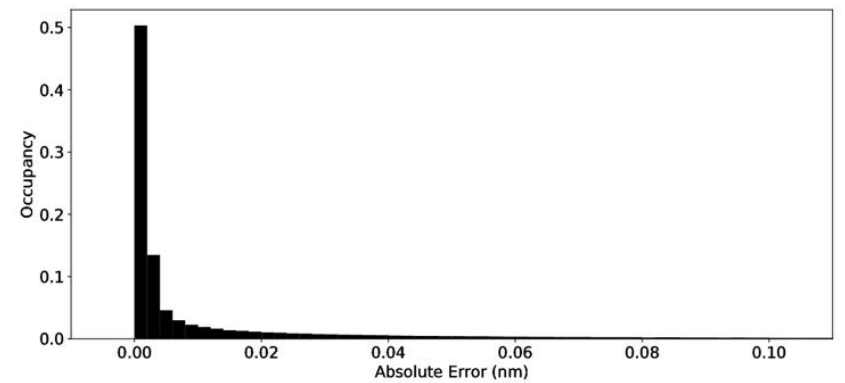

Figure S3. (A) The fitting result of the whole CG molecule for each probe. (B) The histogram of fitting absolute errors of all probes in the inverse $L J$ scale. 


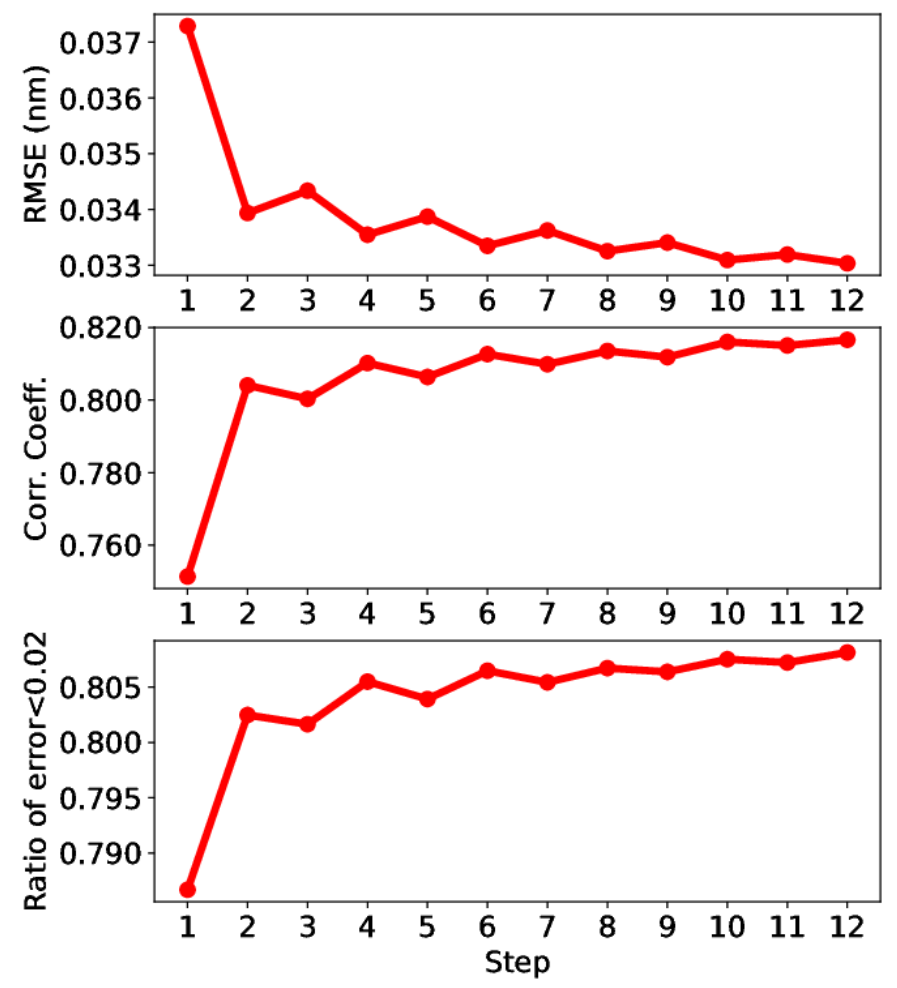

Figure S4. The total fitting error, fitting linear correlation coefficient and ratio of absolute error less than $0.02 \mathrm{~nm}$ with stepwise iterations. 


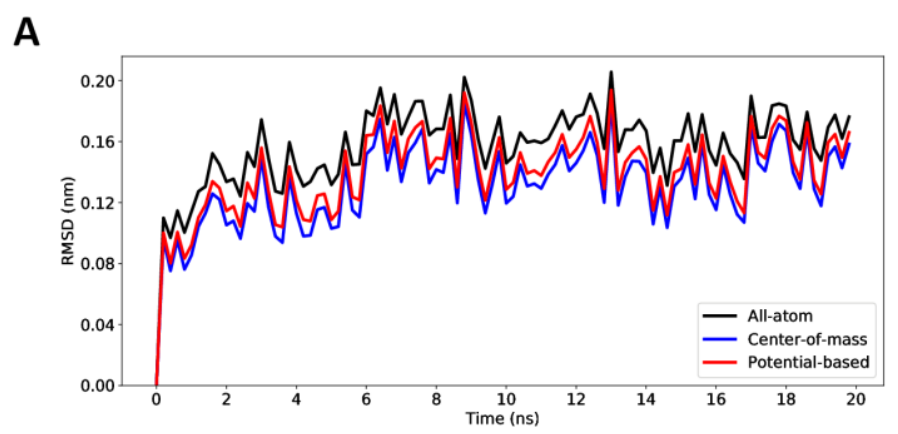

B

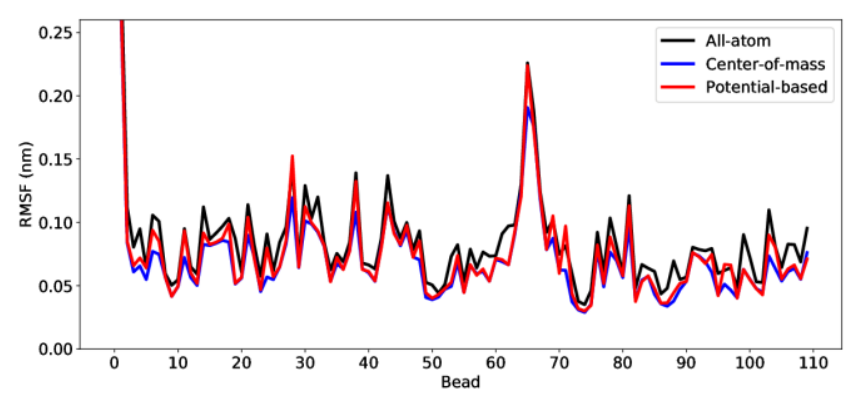

Figure S5. The dynamic property of nonbonded-potential-based mapping compared with centerof-mass mapping and all-atom model. (A) The RMSD of the 20-ns all-atom (AA) trajectory (black), its center-of-mass (COM) mapping trajectory (blue) and its nonbonded-potential-based (NPB) mapping trajectory (red). More than 99\% of NPB mapping RMSD values are closer to AA RMSD values compared with COM to AA. (B) The RMSF of the respective trajectories for each bead (residue). More than $74 \%$ of NPB mapping RMSF values are closer to AA RMSF values compared with COM to AA. 


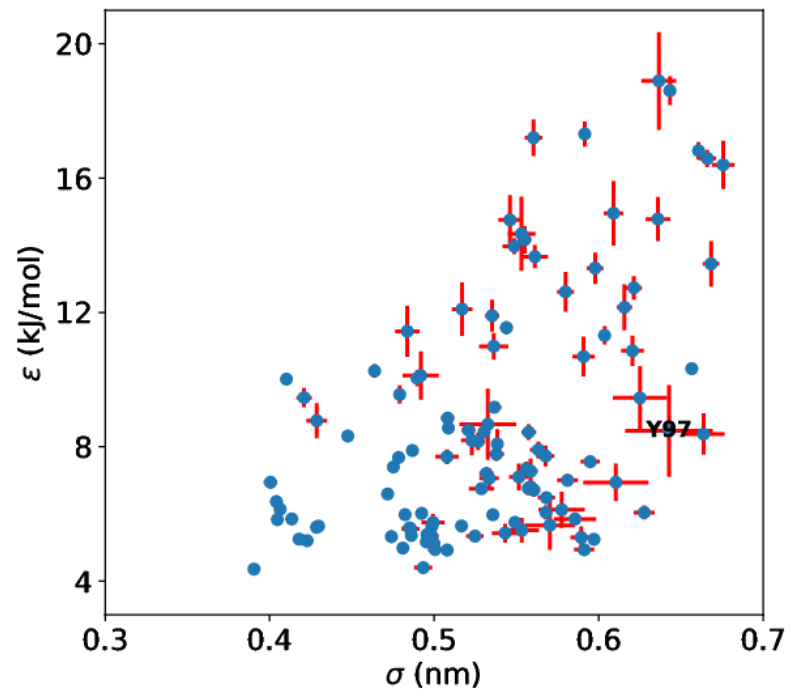

Figure S6. The fitted values of $L J$ parameters $\varepsilon$ and $\sigma$ for each CG bead and its variances among five independent trials. 


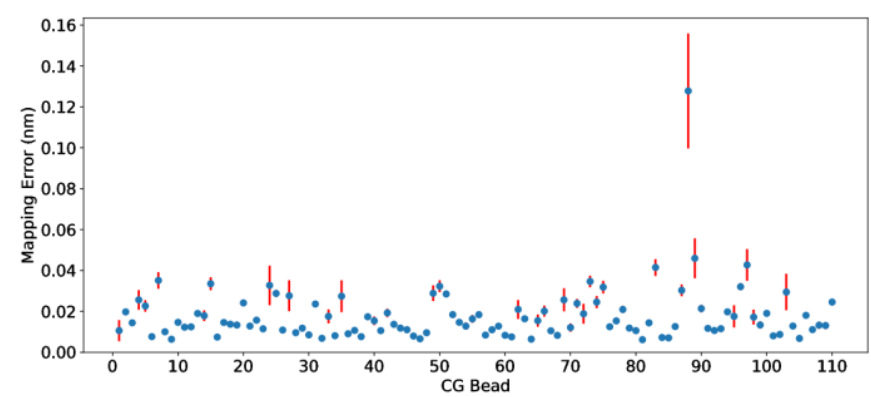

Figure S7. Nonbonded-potential-based mapping error for each CG bead and its variances among five independent trials. 

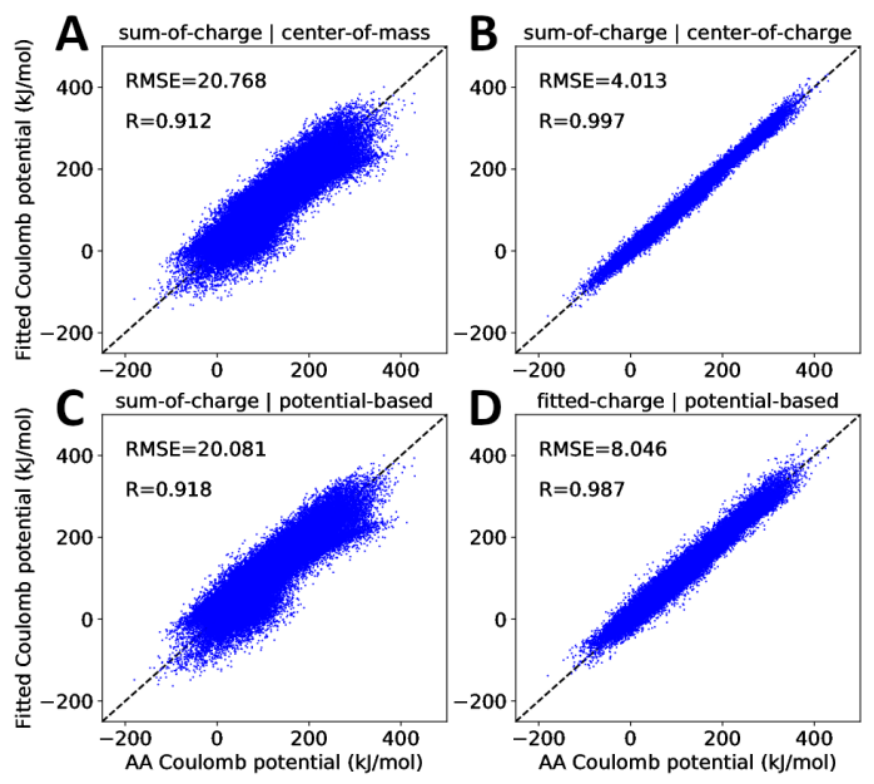

Figure 58. The results of Coulomb potentials subject to different Coulomb parameters together with different mapping functions. (A) Sum-of-charge parameters with center-of-mass mapping. (B) Sum-of-charge parameters with center-of-charge mapping. (C) Sum-of-charge parameters with nonbonded-potential-based mapping. (D) Fitted parameters with nonbonded-potential-based mapping. 


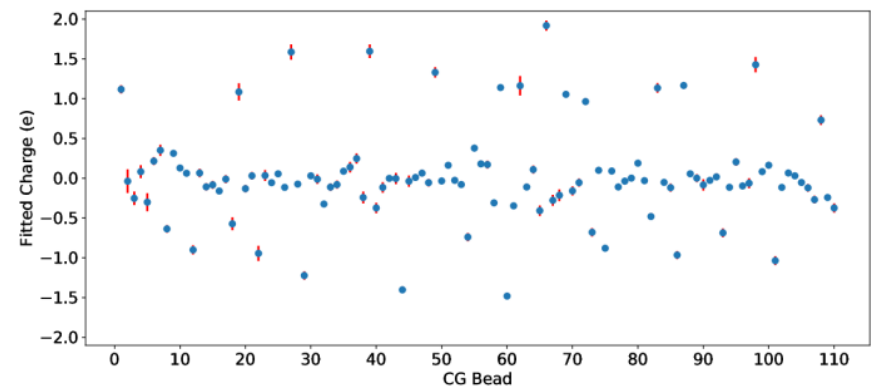

Figure S9. The fitted values of Coulomb parameters $q$ for each CG bead and its variances among five independent trials. 

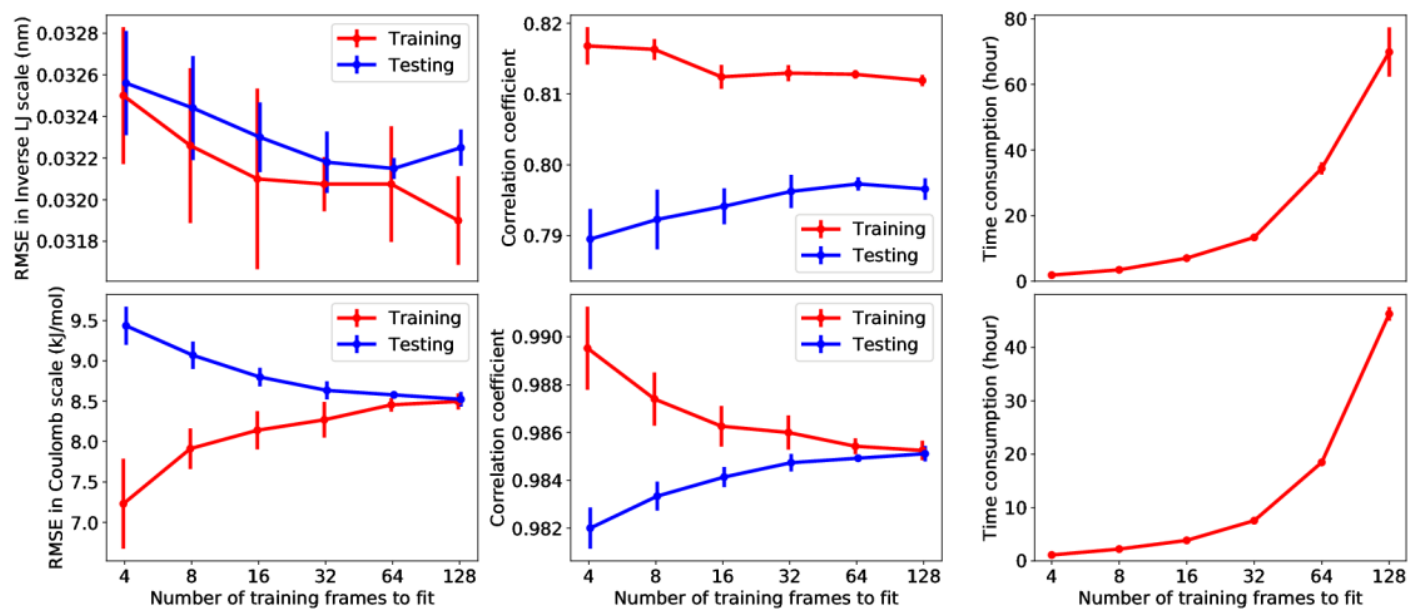

Figure S10. The results of fitting LJ parameters (upper row) and Coulomb parameters (bottom row) using a series of numbers of training frames. The fitting error (left column), fitting linear correlation coefficient (middle column) and computational time consumption (right column) are illustrated. Red for training sets and blue for testing sets. 

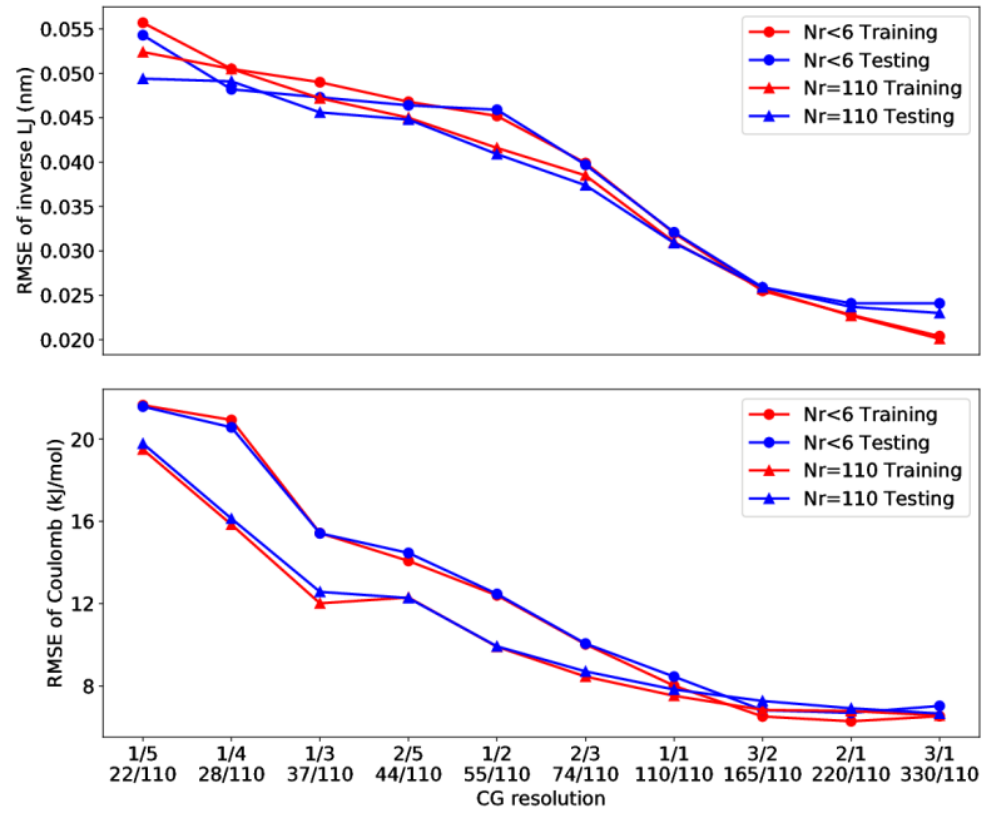

Figure S11. The RMSE of fitting LJ parameters (upper) and Coulomb parameters (bottom) in different CG resolutions. Red for training sets and blue for testing sets. Circle markers for the CG resolutions $1 / 5,1 / 4,1 / 3,2 / 5,1 / 2,2 / 3,1 / 1,3 / 2,2 / 1,3 / 1$ and triangle markers for the CG resolutions 22/110, 28/110, 37/110,44/110, 55/110, 74/110, 110/110, 165/110, 220/110, $330 / 110$. 
A

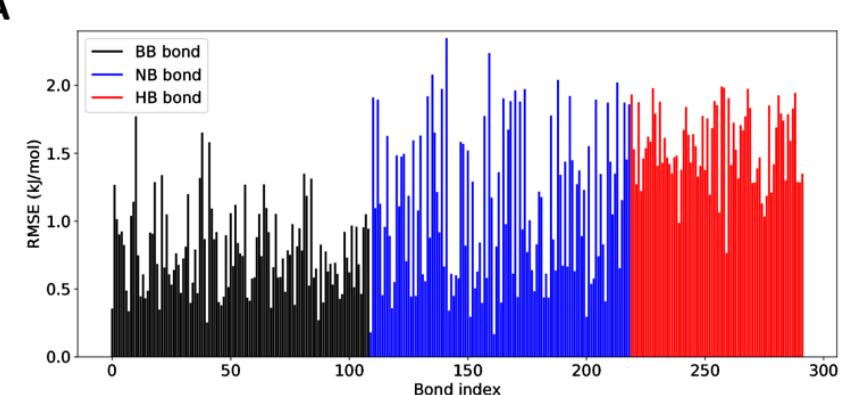

B

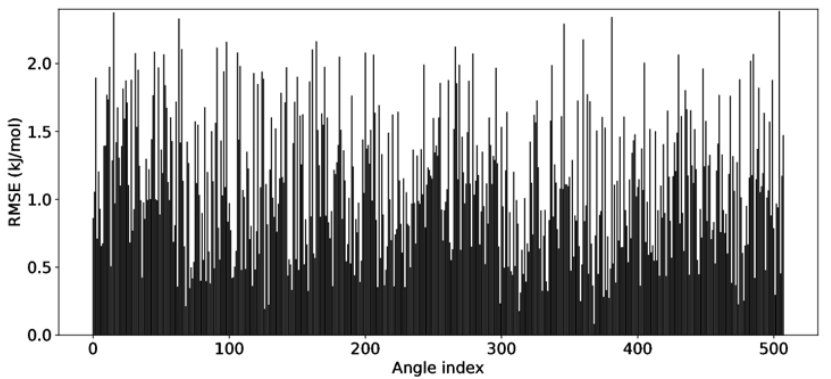

C

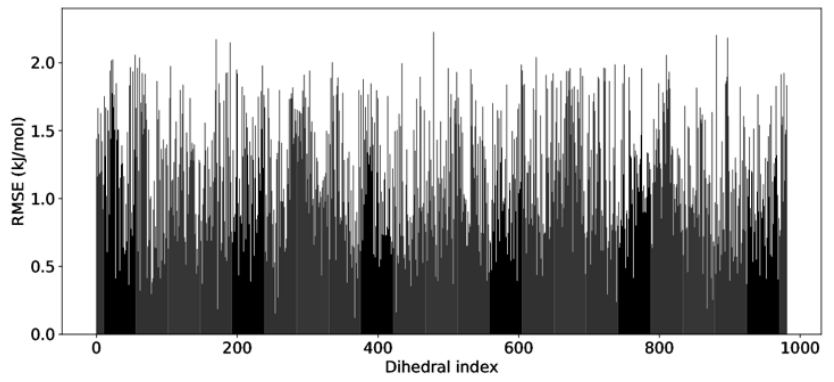

Figure S12. The RMSE of fitting bonded parameters for each (A) bond, (B) angle and (C) dihedral. 
A

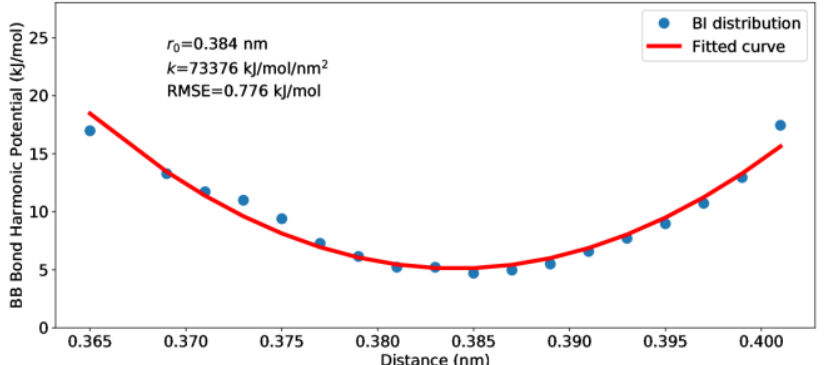

B

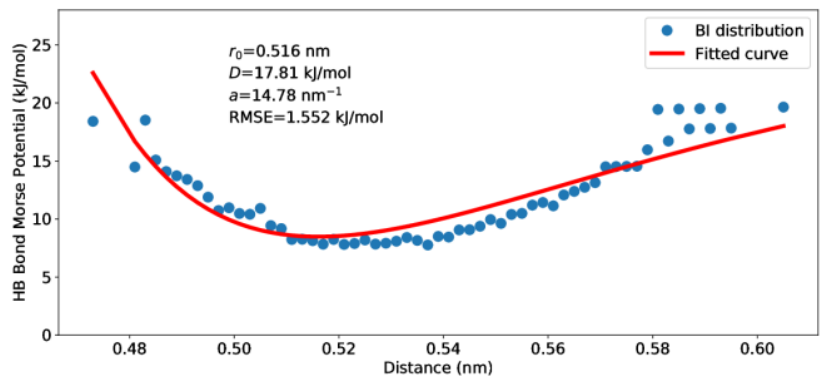

C

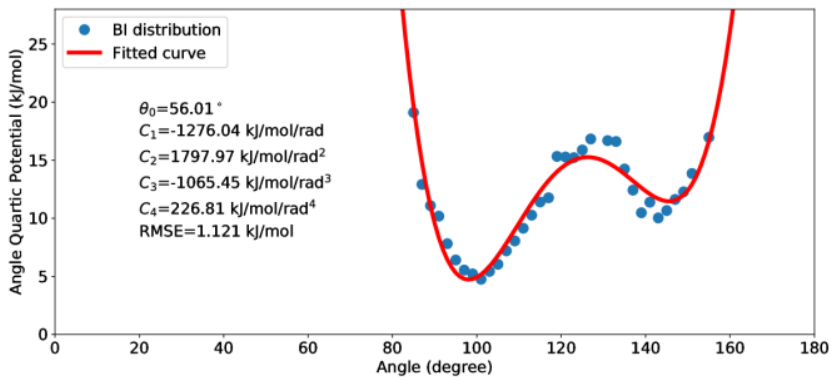

D

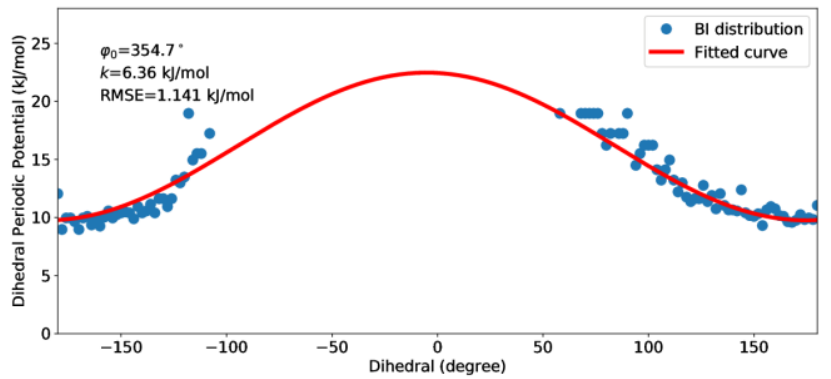

Figure S13. Representatives (closest to average) of fitting bonded parameters. (A) Bond index 90 fitting BB bond harmonic potential. (B) Bond index 246 fitting HB bond Morse potential. (C) Angle index 428 fitting angle quartic potential. (D) Dihedral index 488 fitting dihedral periodic potential. Blue dots are for Boltzmann-inversed distribution in the trajectory and red lines are for the fitted potential curve. 


\section{A}

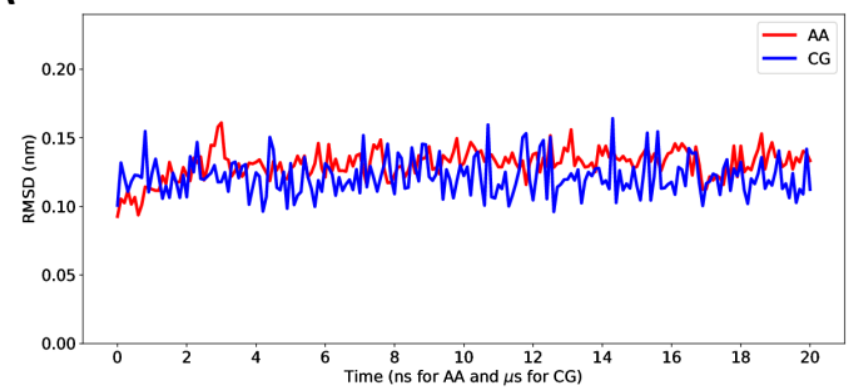

B

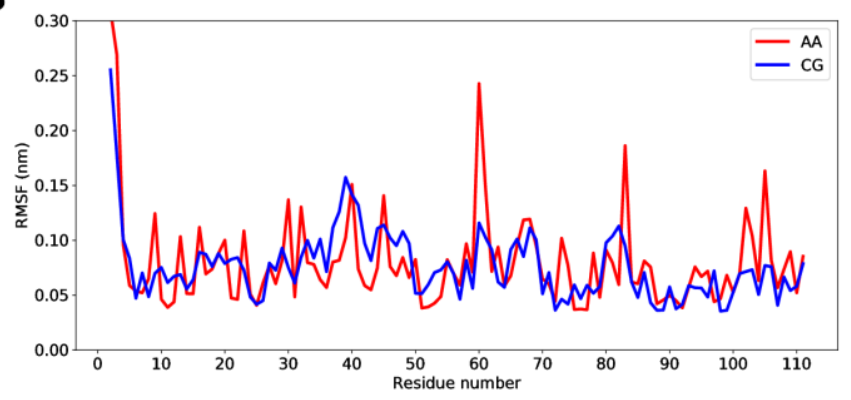

Figure S14. (A) RMSD of 20-ns AA and 20- $\mu$ S CG simulation trajectory aligned to the experimental structure. (B) RMSF of AA and CG simulation. 


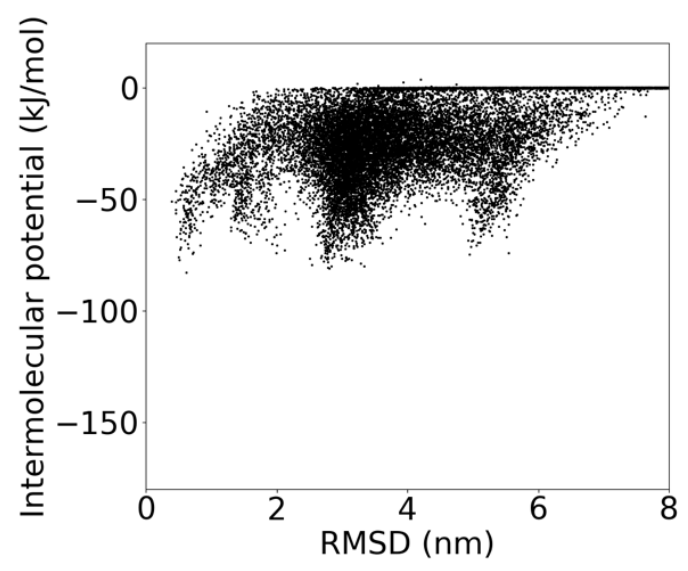

Figure S15. For HLA-KK10m $\times 20$ system, intermolecular potential for each HLA-KK10m complex conformation and its RMSD of KK10m with alignment of HLA in all simulation trajectories. 

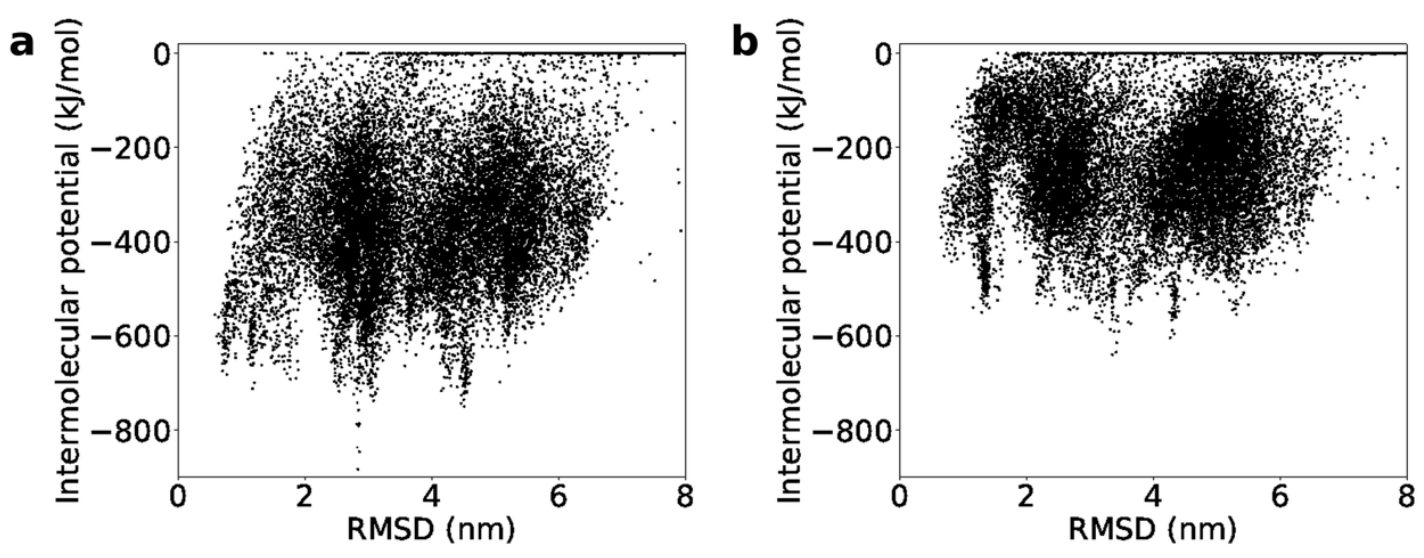

Figure S16. For MARTINI (a) HLA-KK10×20 and (b) HLA-KK10m $\times 20$ system, intermolecular potential for each HLA-KK10 $(\mathrm{m})$ complex conformation and its RMSD of $K K 10(\mathrm{~m})$ with alignment of HLA in all simulation trajectories. 

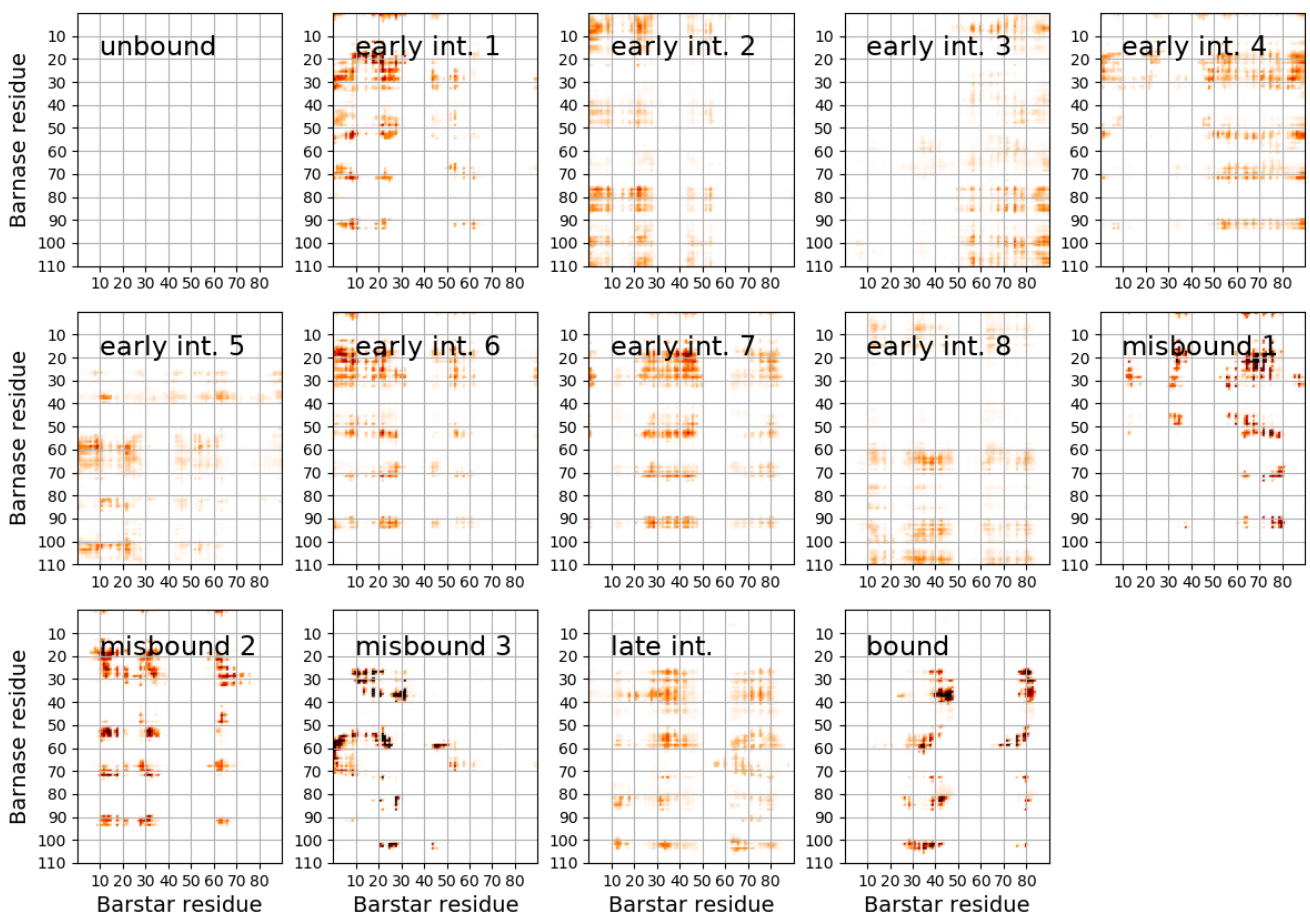

Figure S17. Contact maps of metastable states. Color depth is proportional to contact probability. This figure can be compared with the Figure $\mathrm{S} 3$ of reference ${ }^{19}$. This bound state agrees with the tightly-bound, loosely-bound, pre-bound states in the reference. This late intermediate state agrees with the late intermediate state 1 and 2 in the reference. This unbound state agrees with the unbound state 1 and 2 in the reference ${ }^{19}$. 


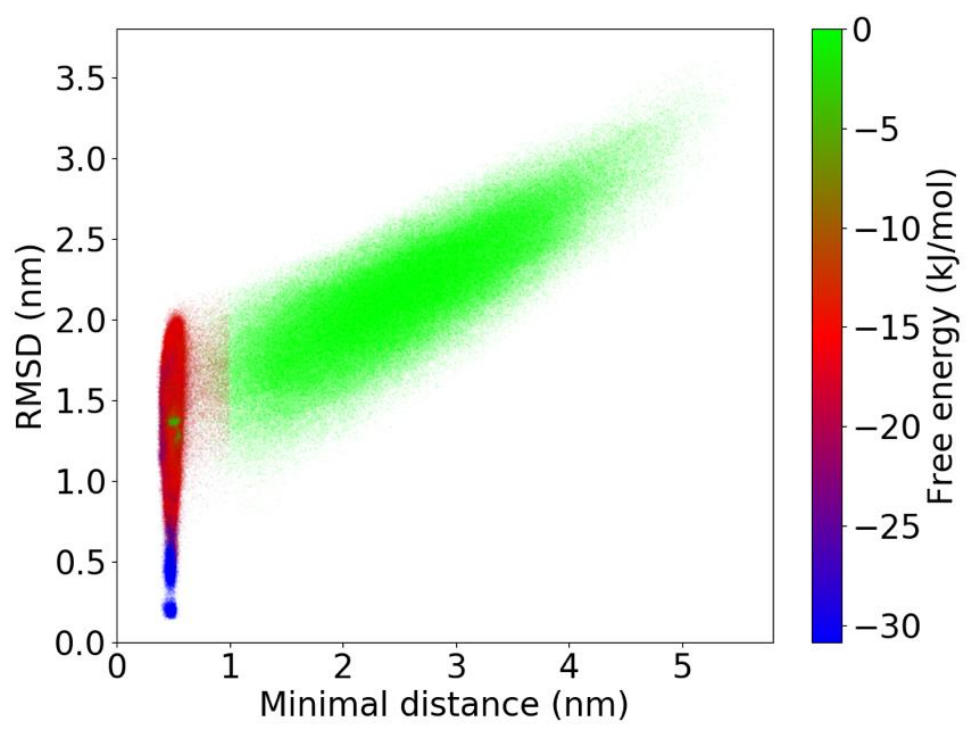

Figure S18. Binding free energy of barnase-barstar conformations in all simulations. The free energy value is estimated by Markov stationary distributions of each metastable state. Minimal distance is the minimum distance between barnase and barstar. RMSD refers RMSD of barnasebarstar complex to the experimental structure. This figure can be compared with Figure $2 \mathrm{c}$ of reference ${ }^{19}$. 


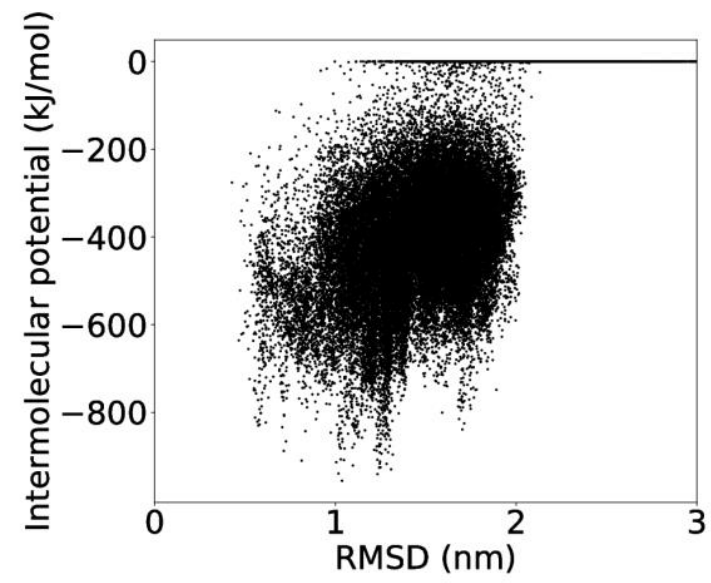

Figure S19. For MARTINI barnase-barstar system, intermolecular potential for each barnasebarstar complex conformation and its RMSD to the experimental structure in all simulation trajectories. 

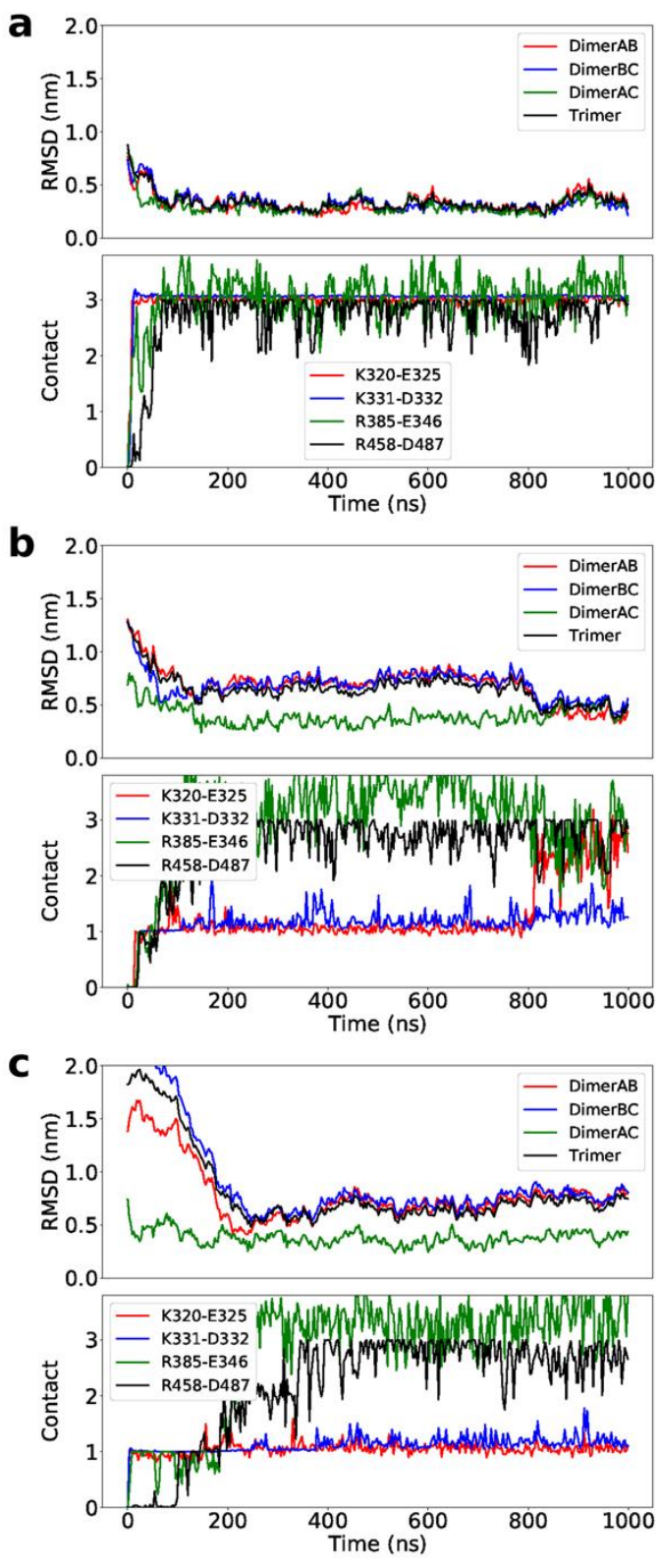

Figure S20. Three trajectories that protomers self-assemble to a (a) native or (b-c) pre-native trimer. (Upper) RMSD of the trimer and three dimers to the experimental structure respectively. (Bottom) contact of the four salt bridges. TRAF2 system is a homotrimer, the three protomers are equivalent, protomer index $A, B, C$ is just for demonstration. 

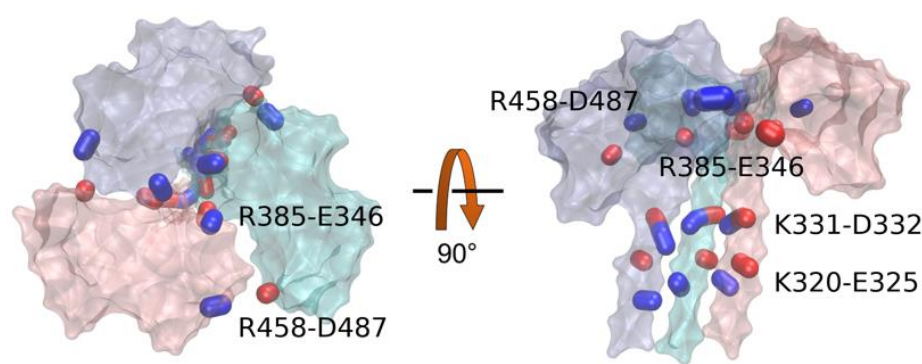

Figure S21. A structural overview of a pre-assembled trimeric TRAF2, which is the conformation at $700 \mathrm{~ns}$ in Figure $5 \mathrm{c}$. Four representative pairs of intermolecular salt bridges at each interface are labeled as solid rods. Four salt bridges are K320-E325 (bottom), K331-D332 (stalk), R385-E346 (topcenter) and R458-D487 (cap edge). Red for acidic residues and blue for basic residues. 


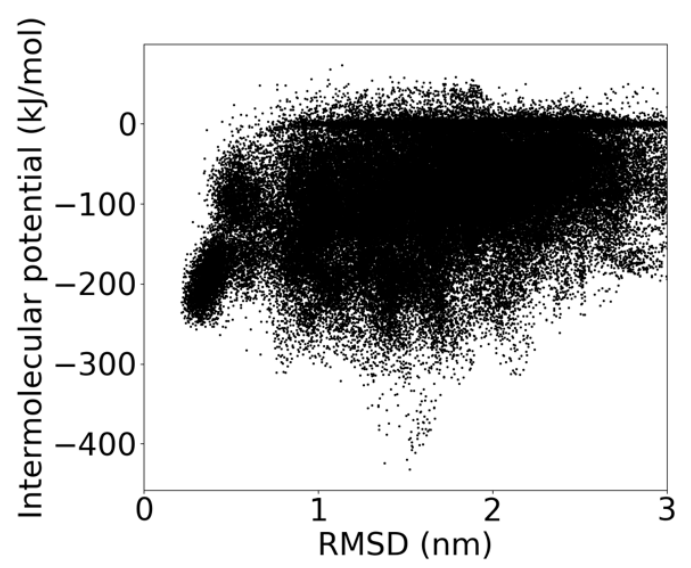

Figure S22. For ART trimeric TRAF2 system, intermolecular potential for each dimer conformation and its RMSD to the dimer of experimental structure in all simulation trajectories. 


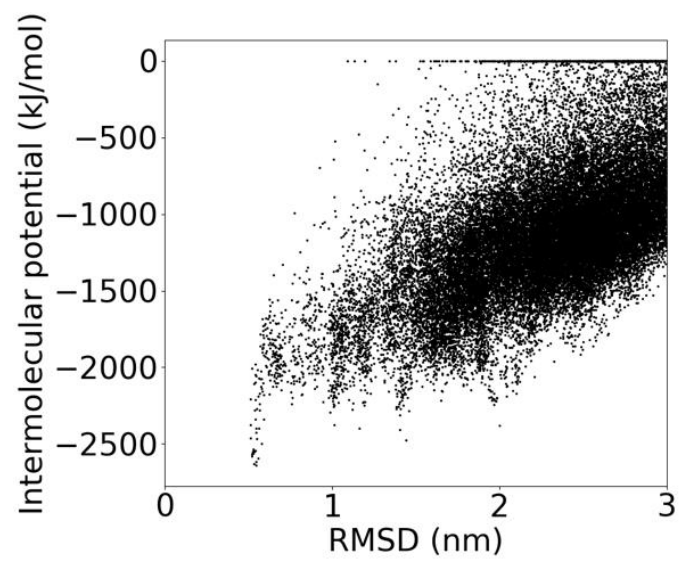

Figure S23. For MARTINI trimeric TRAF2 system, intermolecular potential for each trimer conformation and its RMSD to the experimental structure in all simulation trajectories. 
a

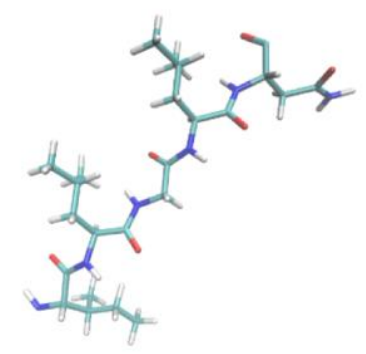

b

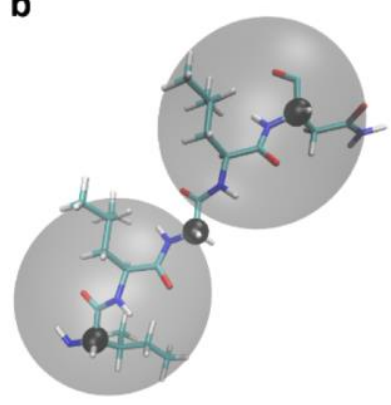

C

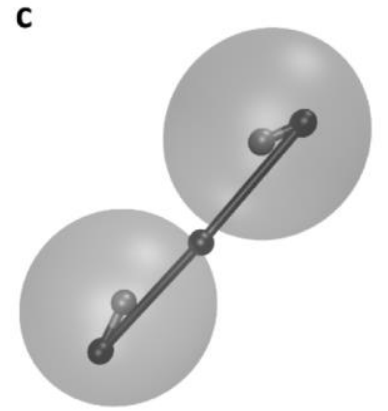

Figure S24. A diagram of two-bead-type CG topology. (a) All-atom representation of a non-capped pentapeptide (ILGLN) as an example. (Red for element O, blue for N, cyan for C, white for $\mathrm{H}$.) (b) Assuming that we choose a CG resolution of 3 BB and 2 NB per 5 residues, then position 3 BB (small black spheres) at the C $\alpha$ of residue 1, 3, 5 and optimize parameters and positions of 2 NB (gray transparent spheres) within the range of the 5 residues. (c) Each BB bonds to its neighbor BB (black bonds); each NB (sites represented by small gray spheres) only bonds to its closest BB (black-gray bonds). 
Supplementary Tables 


\begin{tabular}{|c|c|c|c|c|c|c|}
\hline \multirow{2}{*}{\multicolumn{2}{|c|}{ CG resolution }} & \multicolumn{3}{|c|}{ U } & \multicolumn{2}{|c|}{ Coulomb } \\
\hline & & \multirow{2}{*}{$\frac{\mathrm{RMSE}(\mathrm{nm})}{0.0204}$} & \multirow{2}{*}{$\begin{array}{c}\text { Cor.Coef. } \\
0.9264\end{array}$} & \multirow{2}{*}{$\begin{array}{c}\text { Ratio } \\
0.8645\end{array}$} & \multirow{2}{*}{$\frac{\mathrm{RMSE}(\mathrm{kJ} / \mathrm{mol})}{6.5354}$} & \multirow{2}{*}{$\begin{array}{c}\text { Cor.Coef } \\
0.9912 \\
\end{array}$} \\
\hline $2 / 1$ & training & & & & & \\
\hline I/I & testing & 0.0241 & 0.9080 & 0.8453 & 7.0340 & 0.9899 \\
\hline \multirow{2}{*}{$330 / 110$} & training & 0.0201 & 0.9329 & 0.8661 & 6.5674 & 0.9913 \\
\hline & testing & 0.0230 & 0.9167 & 0.8508 & 6.6665 & 0.9909 \\
\hline \multirow{2}{*}{$2 / 1$} & training & 0.0228 & 0.9170 & 0.8526 & 6.2879 & 0.9918 \\
\hline & testing & 0.0241 & 0.9021 & 0.8487 & 6.6962 & 0.9908 \\
\hline \multirow{2}{*}{$330 / 110$} & training & 0.0227 & 0.9207 & 0.8550 & 6.7879 & 0.9907 \\
\hline & testing & 0.0237 & 0.9031 & 0.8513 & 6.9186 & 0.9902 \\
\hline \multirow{2}{*}{$3 / 2$} & training & 0.0255 & 0.8966 & 0.8425 & 6.5219 & 0.9915 \\
\hline & testing & 0.0259 & 0.8833 & 0.8422 & 6.8298 & 0.9905 \\
\hline \multirow{2}{*}{$165 / 110$} & training & 0.0257 & 0.8979 & 0.8405 & 6.8434 & 0.9908 \\
\hline & testing & 0.0259 & 0.8812 & 0.8428 & 7.2757 & 0.9892 \\
\hline \multirow{2}{*}{$1 / 1$} & training & 0.0320 & 0.8122 & 0.8170 & 8.0160 & 0.9864 \\
\hline & testing & 0.0321 & 0.7977 & 0.8142 & 8.4570 & 0.9853 \\
\hline \multirow{2}{*}{$110 / 110$} & training & 0.0310 & 0.8328 & 0.8212 & 7.5278 & 0.9887 \\
\hline & testing & 0.0309 & 0.8163 & 0.8220 & 7.8319 & 0.9876 \\
\hline \multirow{2}{*}{$2 / 3$} & training & 0.0399 & 0.6864 & 0.7855 & 10.0281 & 0.9799 \\
\hline & testing & 0.0397 & 0.6776 & 0.7858 & 10.0625 & 0.9794 \\
\hline \multirow{2}{*}{$74 / 110$} & training & 0.0385 & 0.7201 & 0.7907 & 8.4615 & 0.9854 \\
\hline & testing & 0.0374 & 0.7056 & 0.8006 & 8.7113 & 0.9844 \\
\hline \multirow{2}{*}{$1 / 2$} & training & 0.0452 & 0.5549 & 0.7681 & 12.4076 & 0.9696 \\
\hline & testing & 0.0459 & 0.5498 & 0.7642 & 12.4748 & 0.9678 \\
\hline \multirow{2}{*}{$55 / 110$} & training & 0.0416 & 0.6505 & 0.7885 & 9.9061 & 0.9795 \\
\hline & testing & 0.0409 & 0.6546 & 0.7889 & 9.9283 & 0.9797 \\
\hline \multirow{2}{*}{$2 / 5$} & training & 0.0468 & 0.5171 & 0.7709 & 14.0806 & 0.9602 \\
\hline & testing & 0.0464 & 0.5146 & 0.7734 & 14.4672 & 0.9564 \\
\hline \multirow{2}{*}{$44 / 110$} & training & 0.0450 & 0.5771 & 0.7740 & 12.3024 & 0.9686 \\
\hline & testing & 0.0448 & 0.5792 & 0.7755 & 12.2764 & 0.9688 \\
\hline \multirow{2}{*}{$1 / 3$} & training & 0.0490 & 0.4887 & 0.7614 & 15.4300 & 0.9509 \\
\hline & testing & 0.0473 & 0.4780 & 0.7730 & 15.4209 & 0.9504 \\
\hline \multirow{2}{*}{$37 / 110$} & training & 0.0472 & 0.5621 & 0.7693 & 12.0200 & 0.9709 \\
\hline & testing & 0.0456 & 0.5589 & 0.7768 & 12.5779 & 0.9673 \\
\hline \multirow{2}{*}{$1 / 4$} & training & 0.0505 & 0.4262 & 0.7613 & 20.9277 & 0.9056 \\
\hline & testing & 0.0482 & 0.4279 & 0.7734 & 20.5704 & 0.9106 \\
\hline \multirow{2}{*}{ 28/110 } & training & 0.0505 & 0.4872 & 0.7613 & 15.8470 & 0.9456 \\
\hline & testing & 0.0491 & 0.4841 & 0.7697 & 16.1357 & 0.9458 \\
\hline 1 & training & 0.0557 & 0.4016 & 0.7352 & 21.6461 & 0.8999 \\
\hline $1 / J$ & testing & 0.0543 & 0.4053 & 0.7435 & 21.5795 & 0.9007 \\
\hline & training & 0.0524 & 0.4382 & 0.7575 & 19.4944 & 0.9168 \\
\hline 2. & testing & 0.0494 & 0.4311 & 0.7736 & 19.7829 & 0.9177 \\
\hline
\end{tabular}


Table S1. The results of fitting $L J$ parameters and Coulomb parameters in different CG resolutions. Similar to Figure $S 4$ for fitting $\sqcup$ parameters and Figure $S 8$ for fitting Coulomb parameters, RMSE represents the total fitting error; Cor.Coef. represents the linear correlation coefficient; Ratio represents the ratio of absolute error less than $0.02 \mathrm{~nm}$. 


\begin{tabular}{|c|c|c|c|}
\hline molecule & PDB & chain & residues \\
\hline \multirow{2}{*}{ HLA } & \multirow{3}{*}{ 4G9D } & $\mathrm{A}$ & 276 \\
\cline { 3 - 4 } & & $\mathrm{B}$ & 99 \\
\cline { 3 - 4 } & & $\mathrm{C}$ & 10 \\
\hline KK10 & & $\mathrm{A}$ & 110 \\
\hline Barnase & \multirow{2}{*}{ 1BRS } & $\mathrm{D}$ & 89 \\
\cline { 1 - 3 } Barstar & & $\mathrm{ABC}$ & $191 \times 3$ \\
\hline TRAF2 & \multirow{2}{*}{ 1CA9 } & $\mathrm{AB}$ & \\
\hline
\end{tabular}

Table S2. The list of experimental structures involved in this work. 


\begin{tabular}{|c|c|c|c|c|}
\hline GPU & CPU cores & \multicolumn{3}{|c|}{ Performance(ns/day) } \\
& Xeon-E5 & AA & MARTINI & ART \\
\hline Tesla-K80 & 12 & 30 & 5524 & \\
\hline Tesla-K20m & 12 & 19 & 5530 & 5533 \\
\hline Tesla-K20m & 4 & & 4686 & 5574 \\
\hline Tesla-K20m & 2 & & 3840 & 4318 \\
\hline Tesla-K20m & 1 & & 2844 & 2831 \\
\hline None & 12 & 7 & 5237 & 7148 \\
\hline None & 4 & & 2625 & 5849 \\
\hline None & 2 & & 1528 & 3840 \\
\hline None & 1 & & 863 & 2491 \\
\hline
\end{tabular}

Table S3. Simulation performance of barnase-barstar system in 10-nm cubic box using GROMACS. The integration timestep used in AA is $2 \mathrm{fs}$, that in MARTINI in $30 \mathrm{fs}$, that in ART is $10 \mathrm{fs}$. 


\section{Supplementary References}

(1) Monticelli, L.; Kandasamy, S. K.; Periole, X.; Larson, R. G.; Tieleman, D. P.; Marrink, S.-J. The MARTINI coarse-grained force field: Extension to proteins. J. Chem. Theory Comput. 2008, $4,819-834$.

(2) Noid, W. G.; Chu, J.-W.; Ayton, G. S.; Krishna, V.; Izvekov, S.; Voth, G. A.; Das, A.; Andersen, H. C. The multiscale coarse-graining method. I. A rigorous bridge between atomistic and coarsegrained models. J. Chem. Phys. 2008, 128.

(3) Cao, Z.; Voth, G. A. The multiscale coarse-graining method. XI. Accurate interactions based on the centers of charge of coarse-grained sites. J. Chem. Phys. 2015, 143.

(4) Ruehle, V.; Junghans, C.; Lukyanov, A.; Kremer, K.; Andrienko, D. Versatile object-oriented toolkit for coarse-graining applications. J. Chem. Theory Comput. 2009, 5, 3211-3223.

(5) Maier, J. A.; Martinez, C.; Kasavajhala, K.; Wickstrom, L.; Hauser, K. E.; Simmerling, C. ff14SB: Improving the accuracy of protein side chain and backbone parameters from ff99SB. $J$. Chem. Theory Comput. 2015, 11, 3696-3713.

(6) Van der Spoel, D.; Lindahl, E.; Hess, B.; Groenhof, G.; Mark, A. E.; Berendsen, H. J. C. GROMACS: Fast, flexible, and free. J. Comput. Chem. 2005, 26, 1701-1718.

(7) Sali, A.; Blundell, T. L. Comparative protein modeling by satisfaction of spatial restraints. $J$. Mol. Biol. 1993, 234, 779-815.

(8) Jorgensen, W. L.; Chandrasekhar, J.; Madura, J. D.; Impey, R. W.; Klein, M. L. Comparison of simple potential functions for simulating liquid water. J. Chem. Phys. 1983, 79, 926-935.

(9) Essmann, U.; Perera, L.; Berkowitz, M. L.; Darden, T.; Lee, H.; Pedersen, L. G. A smooth particle mesh Ewald method. J. Chem. Phys. 1995, 103, 8577-8593.

(10) Hess, B.; Bekker, H.; Berendsen, H. J. C.; Fraaije, J. LINCS: A linear constraint solver for molecular simulations. J. Comput. Chem. 1997, 18, 1463-1472.

(11) Nose, S. A molecular dynamics method for simulations in the canonical ensemble. Mol. Phys. 1984, 52, 255-268.

(12) Hoover, W. G. Canonical dynamics: Equilibrium phase-space distributions. Phys. Rev. A 1985, 31, 1695-1697.

(13) Parrinello, M.; Rahman, A. Polymorphic transitions in single crystals: A new moleculardynamics method. J. Appl. Phys. 1981, 52, 7182-7190.

(14) Bussi, G.; Donadio, D.; Parrinello, M. Canonical sampling through velocity rescaling. J. Chem. Phys. 2007, 126.

(15) Tironi, I. G.; Sperb, R.; Smith, P. E.; Vangunsteren, W. F. A generalized reaction field method for molecular dynamics simulations. J. Chem. Phys. 1995, 102, 5451-5459.

(16) Noé, F.; Nüske, F. A variational approach to modeling slow processes in stochastic dynamical systems. Multiscale Model. Simul. 2013, 11, 635-655. 
(17) Noé, F.; Wu, H.; Prinz, J.-H.; Plattner, N. Projected and hidden Markov models for calculating kinetics and metastable states of complex molecules. J. Chem. Phys. 2013, 139.

(18) Scherer, M. K.; Trendelkamp-Schroer, B.; Paul, F.; Pérez-Hernández, G.; Hoffmann, M.; Plattner, N.; Wehmeyer, C.; Prinz, J.-H.; Noé, F. PyEMMA2: A software package for estimation, validation, and analysis of Markov models. J. Chem. Theory Comput. 2015, 11, 5525-5542.

(19) Plattner, N.; Doerr, S.; De Fabritiis, G.; Noe, F. Complete protein-protein association kinetics in atomic detail revealed by molecular dynamics simulations and Markov modelling. Nat. Chem. 2017, 9, 1005-1011. 